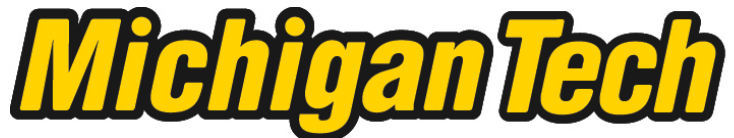 \\ Michigan Technological University Create the Future Digital Commons @ Michigan Tech
}

\section{Utilizing Gaussian-Schell model beams to mitigate atmospheric turbulence in free space optical communications / by Kyle R. Drexler.}

Kyle R. Drexler

Michigan Technological University

Follow this and additional works at: https://digitalcommons.mtu.edu/etds

Part of the Electrical and Computer Engineering Commons

Copyright 2012 Kyle R. Drexler

\section{Recommended Citation}

Drexler, Kyle R., "Utilizing Gaussian-Schell model beams to mitigate atmospheric turbulence in free space optical communications / by Kyle R. Drexler.", Dissertation, Michigan Technological University, 2012.

https://doi.org/10.37099/mtu.dc.etds/79

Follow this and additional works at: https://digitalcommons.mtu.edu/etds

Part of the Electrical and Computer Engineering Commons 


\title{
UTILIZING GAUSSIAN-SCHELL MODEL BEAMS TO MITIGATE ATMOSPHERIC TURBULENCE IN FREE SPACE OPTICAL COMMUNICATIONS
}

\author{
By
}

Kyle R. Drexler

\begin{abstract}
A DISSERTATION
Submitted in partial fulfillment of the requirements for the degree of DOCTOR OF PHILOSOPHY

(Electrical Engineering)
\end{abstract}

MICHIGAN TECHNOLOGICAL UNIVERSITY

2012

(C)2012 Kyle R. Drexler 
This dissertation, "Utilizing Gaussian-Schell Model Beams to Mitigate Atmospheric Turbulence in Free Space Optical Communications," is hereby approved in partial fulfillment of the requirements for the Degree of DOCTOR OF PHILOSOPHY IN ELECTRICAL ENGINEERING.

Department of Electrical and Computer Engineering

Signatures:

Dissertation Advisor

Michael C. Roggemann

Department Chair

Daniel R. Fuhrmann

Date 
To my family and friends. 


\section{Contents}

List of Figures $\ldots \ldots \ldots \ldots \ldots \ldots \ldots \ldots \ldots$ ix

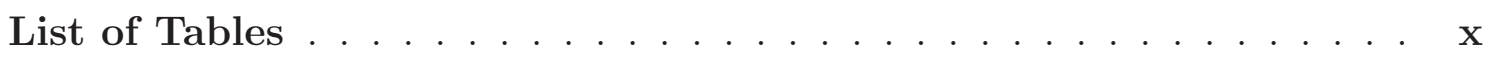

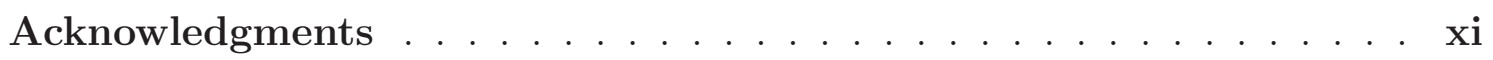

List of Abbreviations \& Symbols . . . . . . . . . . . . xii

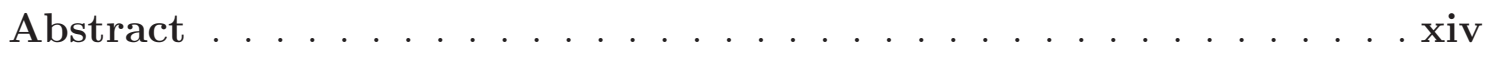

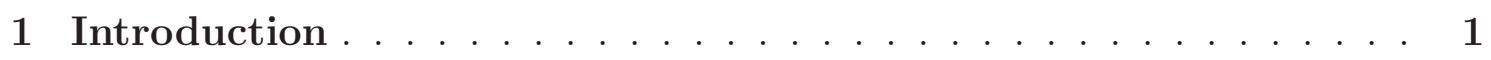

1.1 Traditional Free Space Optical Communications . . . . . . . . . . . 1

1.2 Partial Coherence Approach . . . . . . . . . . . . . . . . . 2

1.3 Summary of Key Results . . . . . . . . . . . . . . . . . 3

1.3.1 Gaussian-Schell Theory and Experiment . . . . . . . . . 3

1.3.2 Optimizing the Gaussian-Schell Model . . . . . . . . . . . 4

1.3.3 Minimum Spatial Light Modulator Resolution . . . . . . . 5

1.4 Dissertation Overview . . . . . . . . . . . . . . . 5

2 Gaussian-Schell Model of Partial Coherence: Theory and Exper-

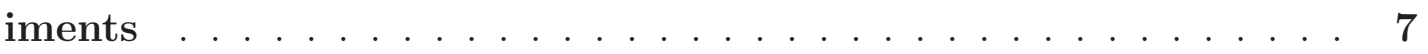

2.1 Introduction . . . . . . . . . . . . . . 7

2.2 Theory . . . . . . . . . . . . . . . . . . . 10 
2.3 Simulation . . . . . . . . . . . . . . . . . . 15

2.4 Experiment . . . . . . . . . . . . . . . . . . 20

2.4 .1 Setup . . . . . . . . . . . . . 20

2.4 .2 Results. . . . . . . . . . . . . . . . . . 23

2.5 Conclusion and Future Work . . . . . . . . . . . . . . 25

3 Upper Coherence Limit _ . . . . . . . . . . . . . . . . . . . . . 35

3.1 Introduction . . . . . . . . . . . . . . . . . 35

3.2 Theory . . . . . . . . . . . . . . . . . . . 37

3.3 Simulation . . . . . . . . . . . . . . . . . . . . . 40

3.3 .1 Channel Description . . . . . . . . . . . . . . 40

3.3 .2 Constants . . . . . . . . . . . . . . . . . . 41

3.3.2.1 Small Aperture Model . . . . . . . . . . . . . . . 41

3.3.2.2 Large Aperture Model . . . . . . . . . . . . . . . 41

3.3 .3 Analysis . . . . . . . . . . . . . . . . . 4 42

3.4 Conclusions . . . . . . . . . . . . . . . . . . . . . . . 49

4 A Technique for Estimating the Number of Control Elements needed to Approximate the Gaussian-Schell model in a Free Space Optical Communications Channel . . . . . . . . . . . . . 52

4.1 Introduction . . . . . . . . . . . . . . . . . 52

4.2 Mathematical Model . . . . . . . . . . . . . . . . . 53

4.3 Simulation Results . . . . . . . . . . . . . . . . . . . 59

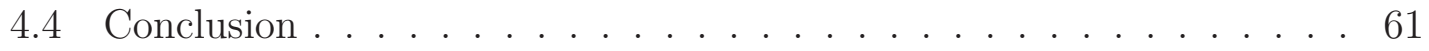

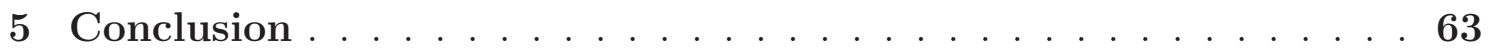

5.1 Summary . . . . . . . . . . . . . . . . . 63 
5.2 Future Work . . . . . . . . . . . . . . . . . . . 64

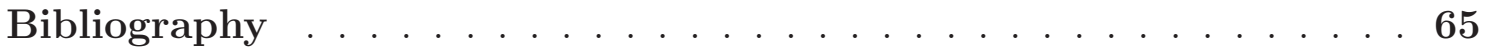

Index . . . . . . . . . . . . . . . . . . . . . . 69 


\section{List of Figures}

2.1 Simulation Setup $\ldots \ldots \ldots \ldots \ldots \ldots$

2.2 Cross sections of beam total as imaged in the far-field . . . . . . . . 18

2.3 Center pixel intensity comparison through turbulence. . . . . . . . . 19

2.4 Center pixel standard deviation and mean over 156 different turbulence screens. . . . . . . . . . . . . . . . . . 20

2.5 Scintillation Index over 156 different turbulence screens for each Fried parameter. . . . . . . . . . . . . . . . . . . 21

2.6 Optical Bench Setup . . . . . . . . . . . . . . . . . . . . . . 22

2.7 Beam Comparison: GSM vs FCS $(\mathscr{S}=8.35) \ldots \ldots \ldots \ldots$

2.8 Beam Comparison: GSM vs FCS $(\mathscr{S}=11.7) \ldots \ldots \ldots \ldots$

2.9 Beam Comparison: GSM vs FCS $(\mathscr{S}=15.7) \ldots \ldots \ldots \ldots$

2.10 Beam Comparison: GSM vs FCS $(\mathscr{S}=20.2) \ldots \ldots \ldots \ldots$

2.11 Beam Comparison: GSM vs FCS $(\mathscr{S}=25.3) \ldots \ldots \ldots \ldots$

2.12 Beam Comparison: GSM vs FCS $(\mathscr{S}=31.0) \ldots \ldots \ldots$

2.13 Beam Comparison: GSM vs FCS $(\mathscr{S}=37.2) \ldots \ldots \ldots$

2.14 Radial average beam comparison of a partially spatial coherent beam to a coherent LASER source. Turbulence strength of $\mathscr{S}=8.35$. . . 29

2.15 Radial average beam comparison of a partially spatial coherent beam to a coherent LASER source. Turbulence strength of $\mathscr{S}=11.7 . \ldots 30$ 
2.16 Radial average beam comparison of a partially spatial coherent beam to a coherent LASER source. Turbulence strength of $\mathscr{S}=15.7$. . . 30

2.17 Radial average beam comparison of a partially spatial coherent beam to a coherent LASER source. Turbulence strength of $\mathscr{S}=20.2$. . . . 31

2.18 Radial average beam comparison of a partially spatial coherent beam to a coherent LASER source. Turbulence strength of $\mathscr{S}=25.3$. . . . 31

2.19 Radial average beam comparison of a partially spatial coherent beam to a coherent LASER source. Turbulence strength of $\mathscr{S}=31.0$. . . . 32

2.20 Radial average beam comparison of a partially spatial coherent beam to a coherent LASER source. Turbulence strength of $\mathscr{S}=37.2$. . . . 32

2.21 Calculated scintillation index reduction data for $200(x, y)$ coordinates near the center of the receiver for each turbulence strength. . . 33

2.22 Histogram of the scintillation index reduction data in Figure 2.21. . . 33

2.23 Spread of the calculated scintillation index reduction over the entire receiver for each turbulence strength. There are 307,200 calculated values for each turbulence strength. . . . . . . . . . . . . . . . 34

2.24 Histogram of the scintillation index reduction data in Figure 2.23. . . 34

3.1 Free Space Optical Communications Channel Overview . . . . . . . . 40

3.2 Average received irradiance of increasing the spatial coherence of the GSM compared to a FCS through turbulence. [Small Aperture Model with $\left.r_{0}=0.3\right] \ldots \ldots \ldots \ldots$. . . . . . . . . . . . . . 42

3.3 Average received irradiance of increasing the spatial coherence of the GSM compared to a FCS through turbulence. [Small Aperture Model

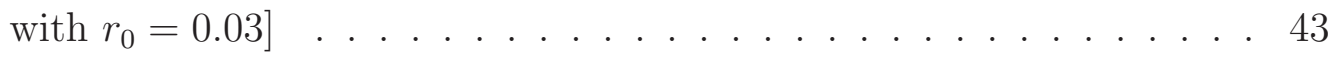


3.4 Average received irradiance of increasing the spatial coherence of the GSM compared to a FCS through turbulence. [Small Aperture Model with $\left.r_{0}=0.003\right] \ldots \ldots \ldots \ldots \ldots \ldots \ldots$

3.5 Spatial statistics: GSM vs FCS $\left(r_{0}=0.3\right) \ldots \ldots \ldots \ldots$

3.6 Spatial Statistics: GSM vs FCS $\left(r_{0}=0.03\right) \ldots \ldots \ldots \ldots$

3.7 Spatial Statistics: GSM vs FCS $\left(r_{0}=0.003\right) \ldots \ldots \ldots \ldots$

3.8 Scintillation Index of GSM. [Small Aperture Model] . . . . . . . . . . 48

3.9 Scintillation Index of GSM. [Small Aperture Model] . . . . . . . . . 49

3.10 Scintillation Index of GSM. [Small Aperture Model] . . . . . . . . . 50

3.11 Scintillation Index of GSM. [Large Aperture Model] . . . . . . . . . 50

3.12 Scintillation Index of GSM. [Large Aperture Model] . . . . . . . . . . 51

4.1 Overview of split-step simulation method for one instance of the GSM. 54

4.2 Phase Screen Sampling Details . . . . . . . . . . . . . . . 56

4.3 (Left) Physical comparison of SLM and the atmospheric channel. (Right) Simulated SLM and atmospheric channel overlay at $z=0 . .57$

4.4 Overview of split-step simulation method to fully develop the GSM. . 58

4.5 Scintillation Index vs Controllable Pixel Elements, Fried Parameter $=0.3 \mathrm{~m} \ldots \ldots \ldots \ldots \ldots \ldots \ldots \ldots \ldots \ldots \ldots \ldots \ldots \ldots \ldots \ldots$

4.6 Scintillation Index vs Controllable Pixel Elements, Fried Parameter

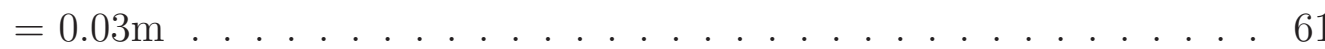

4.7 Scintillation Index vs Controllable Pixel Elements, Fried Parameter $=0.009 \mathrm{~m} \ldots \ldots \ldots \ldots \ldots \ldots \ldots \ldots \ldots \ldots \ldots \ldots \ldots \ldots \ldots \ldots$ 


\section{List of Tables}

2.1 Variance analysis of received intensity data . . . . . . . . . . . . . 24 


\section{Acknowledgments}

I would like to thank my adviser, Dr. Roggemann, for all the support and guidence that he has given me during my tenure at Michigan Tech. 


\title{
List of Abbreviations \& Symbols
}

\author{
$\Delta_{a t m o}$ Atmospheric sampling \\ $\lambda \quad$ Source Wavelength \\ $\mathscr{F} \quad$ Fourier Transform \\ $\mathscr{F}^{-1}$ Inverse Fourier Transform \\ $\mathscr{T} \quad$ Turbulence Phase Screen \\ $\theta(x, y)$ Phase Control Screen \\ $C_{n}^{2} \quad$ Atmospheric Structure Constant \\ $E(x, y)$ Inital amplitude Gaussian Beam \\ $f_{l} \quad$ Focal Length \\ H Fresnel Propagator \\ $k \quad$ Scalar Wavenumber \\ $L_{a t m o}$ Phyiscal length of the atmospheric boundary edge \\ $L_{s l m}$ Physical length of the spatial light modulator edge \\ $r_{0} \quad$ Fried parameter
}


SI Scintillation Index

$S I_{d B}$ Scintillation Index measured in decibels.

$w_{0} \quad$ Beam Waist

z Total Propagation Distance

FCS Fully Coherent Source

FSO Free Space Optical

GSM Gaussian-Schell Model

K Kelvins

L Physical Array length

P Pressure

PCB Partially Coherent Beam

PSD Power Spectral Density

RF Radio Frequency

SLM Spatial Light Modulator

SNR Signal-to-Noise Ratio

T Temperature

UCL Upper-Coherence Limit 


\section{Abstract}

Turbulence affects traditional free space optical communication by causing speckle to appear in the received beam profile. This occurs due to changes in the refractive index of the atmosphere that are caused by fluctuations in temperature and pressure, resulting in an inhomogeneous medium. The Gaussian-Schell model of partial coherence has been suggested as a means of mitigating these atmospheric inhomogeneities on the transmission side.

This dissertation analyzed the Gaussian-Schell model of partial coherence by verifying the Gaussian-Schell model in the far-field, investigated the number of independent phase control screens necessary to approach the ideal Gaussian-Schell model, and showed experimentally that the Gaussian-Schell model of partial coherence is achievable in the far-field using a liquid crystal spatial light modulator. A method for optimizing the statistical properties of the Gaussian-Schell model was developed to maximize the coherence of the field while ensuring that it does not exhibit the same statistics as a fully coherent source. Finally a technique to estimate the minimum spatial resolution necessary in a spatial light modulator was developed to effectively propagate the Gaussian-Schell model through a range of atmospheric turbulence strengths.

This work showed that regardless of turbulence strength or receiver aperture, transmitting the Gaussian-Schell model of partial coherence instead of a fully coherent source will yield a reduction in the intensity fluctuations of the received field. By measuring the variance of the intensity fluctuations and the received mean, it is shown through the scintillation index that using the Gaussian-Schell model of partial coherence is a simple and straight forward method to mitigate atmospheric turbulence instead of traditional adaptive optics in free space optical communications. 


\section{Chapter 1}

\section{Introduction}

\subsection{Traditional Free Space Optical Communica- tions}

In traditional free space optical (FSO) communications a fully coherent source, commonly known as a LASER, is propagated through the atmosphere to a receiver. Due to changes in temperature and pressure, the atmosphere is inherently inhomogeneous, causing the index of refraction to fluctuate resulting in intensity fluctuations of the propagating field at the receiver. This phenomena is commonly referred to as

speckle. ${ }^{1-6}$ To compensate for these fluctuations expensive adaptive optics solutions are generally implemented to measure and correct the wave front of the propagating field. ${ }^{7}$

The two most common adaptive optics methods for measuring atmospheric disturbances are the Hartmann wavefront sensor and the Shearing Interferometer wavefront sensor ${ }^{7}$. Both of these methods compensate by measuring the slope of the propagating wave front phase. When these devices are implemented in a FSO system there are generally two different sensors, one to measure the wavefront aberra- 
tions and another to collect the desired information. To implement this system in real time a deformable mirror is used before the data sensor to compensate for the measured wave front aberrations. If real time processing is not required this can be delegated to post-processing. To reduce receiver complexity in a FSO system mitigating these fluctuations on the transmission side is preferable.

\subsection{Partial Coherence Approach}

Transmitting a partially coherent source has been suggested as a means to mitigate

these intensity fluctuations and reduce the sensor complexity of a FSO system. ${ }^{6,8-17}$ Specifically this dissertation analyzes the Gaussian-Schell model ${ }^{18,19}$ (GSM) of partial coherence to mitigate atmospheric turbulence effects in a horizontal free space optical communications channel. A horizontal atmospheric channel was chosen to simplify the mathematics of atmospheric propagation and shorten simulation times. In this approach for generating the $\mathrm{GSM}^{9}$, different independently generated phase control screens are generated and applied to an initial amplitude Gaussian beam to statistically control the phase of the propagating beam allowing for optimal transmission $^{11}$. Each independent phase control screen after being applied to the Gaussian beam and propagated will be known as a single instance of the GSM. When they are all combined at the receiver over a specified averaging time they will then yield a fully developed GSM.

Different methods to generate partial coherence have been attempted in the past with varying degrees of success. One of the most basic methods uses a rotating phase diffuser to reduce the spatial coherence of the source ${ }^{20}$. The problem with this method is that the spatial coherence characteristics are directly related to the phase diffuser, and changing the beam properties is not possible without creating 
another diffuser. The other problem with rotating phase diffusers is that the phase pattern will repeat itself each time the diffuser rotates causing an unwanted temporal correlation in the random pattern leading to a reduction in the effectiveness of the approach. Another suggested method uses a multi-mode laser where the coherence is dependent upon the lack of phase correlation between modes ${ }^{21}$. The technical issue here is in controlling the degree of partial coherence. To overcome these problems an adaptive phase control device is desirable.

A previous adaptive method that was successful in creating partial coherence used a deformable membrane mirror with 37 addressable actuators. ${ }^{10}$ The main problem with this approach was that its coherence properties were limited by the perturbation voltages applied to the mirror that followed a Bernoulli distribution. The difference between the previous adaptive method and this system is a fully

controllable liquid crystal spatial light modulator (SLM) will be used for the experiments and subsequently modeled in simulation. The benefits of this system allow any type of phase screen to be applied, yielding a greater number of partially coherent states. This increased number of states allows better control of the beam which results in better transmission optimization.

\subsection{Summary of Key Results}

\subsubsection{Gaussian-Schell Theory and Experiment}

This dissertation details the theory of partial coherence and how it applies to the Gaussian-Schell model (GSM) and then presents a wave optics simulation and experiment to prove the concept using a liquid crystal SLM. The wave optics simulation verified the GSM in the far-field and analyzed how many instances of a GSM were needed to approach the ideal GSM ${ }^{18,19}$. This is important because a spatial light 
modulator is currently frame-rate limited, and this information helped to optimize the results while staying within the limitations of the experiment. This work verified the GSM in the far-field, demonstrated that a liquid crystal spatial light modulator is spatially able to implement a GSM in the far-field, and when implemented the calculated scintillation index from the received field is less than a fully coherent source.

\subsubsection{Optimizing the Gaussian-Schell Model}

After verifying that the phase statistics are able to be controlled using a SLM I demonstrate the concept of controlled coherence in a simulated optical communications channel to determine the optimal spatial correlation width of a GSM beam to mitigate turbulence and maintain a high received power. This is done by creating both a partially coherent source and a fully coherent source(FCS) using the SLM, which is achievable by controlling the spatial correlation width of the phase control screen. The larger the spatial correlation width of the phase control screen the more coherent the propagating beam will be, while the inverse is true as well.

By defining a range of spatial correlation widths we are able to transmit a FCS and a range of GSM sources to the receiver. Using this received data we are able to easily compare the scintillation index (SI) of the FCS to the range of GSM sources and determine a value that optimizes transmitted power while ensuring that the transmitting beam does not exhibit the spatial variance statistics of a fully coherent source. This optimized value of the spatial correlation width is defined as the upper coherence limit (UCL) of the GSM beam and ensures that the GSM does not exhibit the speckling statistics of a fully coherent source. Two different atmospheric cross-sections were chosen along with a variety of aperture diameters, to show the robustness of the UCL optimization technique. 


\subsubsection{Minimum Spatial Light Modulator Resolution}

To implement this system in a practical FSO system it is necessary to know the minimum spatial resolution necessary in a spatial light modulator. This is especially true given the slow frame rates of the current generation spatial light modulators in terms of FSO system bandwidth. Determining the minimum resolution allows simplification of the control circuitry and will hopefully yield faster frame rates. It is for this reason that a wave optics simulation was developed to estimate the minimum number of phase control elements necessary in a spatial light modulator to generate a Gaussian-Schell model beam through turbulence. This simulation determined the minimum number of phase control elements necessary to stabilize the scintillation index of the received field. A variety of turbulence strengths and receiver diameters were chosen to show that the numerical analysis is valid over a variety of channel parameters and receiver apertures.

\subsection{Dissertation Overview}

The remainder of this dissertation is organized as follows. Chapters two, three, and four contain previously published or submitted work by the author which has been edited for readability, accuracy, and cohesion of the dissertation. The work in chapter two was published in Optical Engineering and an OSA Technical Digest, the

work in chapter three was published solely in Optical Engineering, and the work in chapter four has been submitted to Optical Engineering and is currently undergoing the peer review process. The rest of the document is arranged in the following manner.

In chapter two the Gaussian-Schell theory of partial coherence along with the related experimental work is covered. Chapter three details the optimization tech- 
nique of the upper-coherence limit. Chapter four analyzes the minimum spatial resolution necessary to develop the GSM through a turbulent atmosphere. The fifth and final chapter wraps up the dissertation with an overview of the significance the completed work including some easy to implement suggestions for future work. 


\section{Chapter 2}

\section{Gaussian-Schell Model of Partial Coherence: Theory and Experiments}

\section{$2.1 \quad$ Introduction}

Free space optical communications systems operate by a laser beam propagating through the atmosphere from the transmitter to the receiver. The transmitted light is generally from a uniform intensity, highly spatially coherent laser beam. When this light passes through atmospheric turbulence its spatial coherence properties are affected by turbulence-induced index of refraction fluctuations along the optical path. The result is that if the combination of turbulence strength and path length are sufficient, a strongly temporal and spatially fluctuating intensity pattern falls upon the receiver aperture ${ }^{6}$. This causes power fluctuations at the receiver which can affect performance. One goal of research in the area of free space optical

communications has been to reduce the variance of the power fluctuations while 
simultaneously maintaining a high mean received power. A proposed method to accomplish this is to transmit a partially spatially coherent beam ${ }^{8,13-15,22-24}$. This chapter presents the theory and an experimental demonstration of this concept.

Creating a partially coherent beam and controlling the coherence attributes can be problematic. Of particular interest in this chapter is the concept of controllable coherence using the Gaussian-Schell model ${ }^{9}$ to allow optimal transmission ${ }^{11}$. One method that has created a partially coherent source was done using a deformable membrane mirror with 37 addressable actuators and use of a heater to mimic turbulence effects ${ }^{10}$. The main problems with this approach is the controlling system, where the coherence parameters were limited by the perturbation voltages applied to the mirror that followed a Bernoulli distribution, and the unknown turbulence statistics of the heater. A less complex method to construct a partially coherent beam is to use a rotating phase diffuser to reduce the spatial coherence of the source $^{20}$. The problem with this method is that the phase pattern repeats itself each time the diffuser rotates, which causes an unwanted temporal correlation in the random pattern and reduces the effectiveness of approach. This method also requires the use of a new diffuser every time different turbulence parameters arise to maintain optimal performance. Another suggested method uses a multi mode laser where the coherence is dependent upon the lack of phase correlation between the modes ${ }^{21}$. The problem with this method is in the technical aspect of controlling the degree of partial coherence. To overcome these problems an adaptive phase control device is desirable.

This chapter describes a new technique based on the use of a liquid crystal spatial light modulator (SLM) to control the phase of a fully coherent beam to create 
a partially spatial coherent beam. While current liquid crystal technology does not have sufficient response time, work is being done to improve the responsiveness of phase modulation devices. A recent example by Love ${ }^{25}$ has seen response times for liquid crystal phase modulators drop to the sub millisecond realm. In this chapter the SLM used is roughly two orders of magnitude slower. The concept requires a coherent beam to be sent to the SLM where a sequence of random phase realizations is rapidly applied to the beam. The resulting beam realizations are then averaged at the receiver owing to the finite temporal bandwidth of the detector. Random phase realizations with virtually any spatial statistics can be placed upon the SLM. Here the SLM is used to generate a Gaussian-Schell model beam ${ }^{9}$. The phase applied to the SLM is composed of a Gaussian kernel, $f(x, y)$, that is convolved with a random matrix, $r(x, y)$. By controlling the $\mathrm{e}^{-1}$ radius of the kernel and the standard deviation of the random matrix, $\sigma_{r}$, it is possible to control the degree of coherence of the outgoing beam. Keep in mind that the SLM will need to operate fast enough to correct for each transmitted bit. Therefore in a FSO communications network the SLM will need to run significantly faster than the data rate. If a standard 2.5 Gbps connection is assumed then the SLM, at a bare minimum, would need to be an order of magnitude faster than current technology will allow at this time. While the response time of both the phase control device and the detector are limited in this experiment these results demonstrate the basic principles of the technique.

The work in this chapter differs from what has been done previously by projecting the outgoing beam to the far-field, and controlling the partial coherence of the outgoing beam with a full 2D Gaussian-Schell model on a high resolution liquid crystal SLM that is passed through a near-field turbulence path with known statistics. Measuring the far-field projection was done to ensure that when the experiment is 
performed the results are diffraction limited.

The simulation and experimental results of this chapter demonstrate that by transmitting a partially spatial coherent beam through a turbulent path the variance of the intensity for a single pixel over multiple turbulence screens will decrease. This variance decrease leads to a reduction of the scintillation index, thus, the signal-tonoise ratio of the received signal can be improved without increasing transmitter power.

The remainder of this chapter is organized as follows. In section 2.2 discusses the mathematical background for turbulence modeling, the computer simulation method and partially coherent beam propagation. In section 2.3 the simulation is discussed and how it applies to the theory. In section 2.4 the experiment setup and main results from this experiment are presented and explained. Finally, in section 2.5 briefly discusses future work and presents the conclusions.

\subsection{Theory}

Turbulence is defined as the condition that exists when fluid motion in the atmosphere becomes unstable and random. When this happens it creates packets of air having different indices of refraction resulting from differences in temperature and pressure. The equation for the index of refraction for the atmosphere is ${ }^{7}$

$$
n(\vec{r}, t)=n_{o}+n_{1}(\vec{r}, t)
$$


where $\vec{r}$ is a three dimensional vector position and $t$ is time, $n_{0}$ is the mean index of refraction of air, and $n_{1}$ is the randomly fluctuating term that is dependent upon pressure and temperature of the atmosphere. $n_{1}$ is defined as ${ }^{7}$

$$
n_{1}=\frac{77.6 P}{T} 10^{-6}
$$

where $P$ is the pressure of the air in millibars and $T$ is the temperature of the air in Kelvins. The Kolmogorov spectrum is used to model the spatial characteristics of atmospheric turbulence. The spatial power spectral density (PSD) of $n_{1}$ for Kolmogorov turbulence is represented by, $\Phi_{n}^{K}(k, z) .{ }^{7}$

$$
\Phi_{n}^{K}(k, z)=0.033 C_{n}^{2}(z) k^{-11 / 3}
$$

where $k$ is the scalar wavenumber, and $C_{n}^{2}$ is the structure constant of the index of refraction fluctuations with units $\mathrm{m}^{-2 / 3}$. For this work $C_{n}^{2}$ is kept constant, which is consistent with horizontal propagation through the atmosphere. A single phase screen is used to model turbulence over the path. The method for controlling the spatial coherence of the propagating beam is now presented.

With regard to this concept, the spatial correlation of the random phase applied by the SLM controls the coherence properties of the propagating field. For the work reported here, a Gaussian correlated random phase function is created by convolving the sampled Gaussian filter, $f(x, y)$ with a matrix of random values, $r(x, y)$. The Gaussian filter is given by

$$
f(x, y)=\frac{1}{2 \pi \sigma_{f}^{2}} \exp \left[-\frac{(y \Delta y)^{2}+(x \Delta x)^{2}}{2 \sigma_{f}^{2}}\right]
$$


where $\sigma_{f}^{2}$ is equal to the $e^{-1}$ radius of the Gaussian filter. With $x=(0,1, \ldots, N-1)$ and $y=(0,1, \ldots, M-1)$, where $N$ and $M$ are equal to the number of adjustable pixels of the SLM in the $x$ and $y$ directions, respectively. $\Delta x$ and $\Delta y$ are the spatial sample spacing in the $x$ and $y$ directions. The random matrix is given by

$$
r(x, y)=\frac{[\gamma(x, y)-0.5] \sigma_{r}}{(\Delta y \Delta x)^{1 / 2}}
$$

where $\gamma$ is a random draw from a random number generator whose output is uniformly distributed on $[0,1]$ and $\sigma_{r}$ is the standard deviation desired for the matrix. The phase screen is now defined by the convolution of these functions

$$
\theta(x, y)=[f(x, y) \otimes r(x, y)](\Delta y \Delta x)
$$

where $\otimes$ represents the two dimensional convolution operator. The convolution for the phase screen is carried out by use of fast Fourier transform based methods ${ }^{26}$. The phase screen, $\theta(x, y)$ is applied directly to the SLM, although mathematically the effect on the incident optical field is a multiplication by the complex exponential $\mathrm{e}^{j \theta(x, y)}$. The spatial correlation width, $\sigma_{g}^{2}$, is the $\mathrm{e}^{-1}$ radius of the correlation function of $\theta(x, y)$ and is given by ${ }^{9}$

$$
\sigma_{g}^{2}=\frac{8 \pi \sigma_{f}^{4}}{\sigma_{r}^{2}}
$$

Physically $\sigma_{g}^{2}$ indicates how smooth $\theta(x, y)$ will be. The smaller $\sigma_{g}^{2}$ is the less coherent the outgoing beam will be. In the real world $\sigma_{r}$ relates to the amplitude variation of the phase screen, and $\sigma_{f}$ is the transverse spatial correlation length. Both of these parameters have the units of length.

The expected outcome from applying these statistical phase variations to the prop- 
agating beam is that as the transmitted field coherence decreases the intensity fluctuations of the observed field will also decrease ${ }^{27}$. This means that when using a partially coherent beam instead of a traditional laser source the variance of the received power fluctuations will have decreased. These fluctuations can be quantified by the scintillation index. The scintillation index is the variance of the received signal normalized by the mean signal and is defined as

$$
S I=\frac{\operatorname{variance}(q)}{\operatorname{mean}(q)^{2}}
$$

where $q$ is used to define the received signal power. In this case the signal is physically defined as the intensity of light received.

For reference the analytic expression for the mean intensity of a Gaussian-Schell model beam in the target plane at distance $z$ is included. ${ }^{8}$

$$
<I(\rho)>=\frac{w_{0}^{2}}{w_{\zeta}^{2}(z)} \exp \left[\frac{-2 \rho}{w_{\zeta}^{2}(z)}\right]
$$

Where $w_{0}$ is the source beam radius, $\rho$ is the sum of the field components, and $w_{\zeta}(z)$ is the $\mathrm{e}^{-1}$ radius of the beam in the presence of turbulence at distance $z$ from the aperture.

$$
w_{\zeta}(z)=w_{0}\left(\hat{r}^{2}+\zeta \hat{z}\right)^{2}
$$

Where $\zeta$ is the global coherence parameter

$$
\zeta=1+\frac{w_{0}^{2}}{\sigma_{g}^{2}}+\frac{2 w_{0}^{2}}{\rho_{0}^{2}}
$$

and $\rho_{0}$ is the coherence length of the spherical wave in turbulence. 


$$
\rho_{0}=0.55 C_{n}^{2} k^{2} z
$$

The parameter $\hat{r}$ characterizes the focusing properties of the transmitting beam, where $\hat{r}<1$ for converging beams, $\hat{r}=1$ for collimated beams and $\hat{r}>1$ for divergent beams. Since an initially collimated beam is assumed, $\hat{r}=1$ and $\hat{z}=$ $z /\left(0.5 k w_{b}^{2}\right)$, where $w_{b}$ is the beam waist at $z=0$. Using the notation of Mandel and Wolf ${ }^{18}$ the $\mathrm{e}^{-1}$ radius of $\langle I(\rho)>$ as a function of $z$ is defined as

$$
\bar{\rho}(z)=\frac{w_{\zeta}(z)}{\sqrt{2}}=\sqrt{2} \sigma \Delta(z)
$$

where

$$
\begin{gathered}
w_{b}^{2}=4 \sigma^{2} \\
\Delta(z)=\left[1+\left(\frac{2 z}{k w_{b} \delta}\right)^{2}\right]^{1 / 2},
\end{gathered}
$$

and

$$
\delta^{2}=\frac{4 \sigma^{2} \sigma_{g}^{2}}{4 \sigma^{2}+\sigma_{g}^{2}}
$$

The Gaussian-Schell beam model used in this chapter was demonstrated in a wave optics simulation by $\mathrm{Xiao}^{9}$; the expected outcome from using this model is that the time averaged beam shape at the receiver will be Gaussian ${ }^{24,28}$. Specific beam parameters can be found in ${ }^{8,24}$ where a more complete theoretical treatment of the effects of the atmosphere on a partially coherent beam can be found. 


\subsection{Simulation}

The purpose of the simulation is to validate the theory and show that transmitting a partially spatial coherent beam will result in a beam falling on the receiver plane which is on average Gaussian, with parameters that can be predicted as shown above. It is also important to obtain an estimate of how many realizations of the random phase screen are necessary to average over to obtain this mean profile. Hence, the simulation data is broken down into four different beam profiles based upon the number of frames that were summed. This information is then applied to the experiment to increase the efficiency of the experiment, and to estimate the rate at which a practical system must operate. It is important to note that this simulation is done in the far-field to correspond to the bench experiment that is discussed in section four as opposed to the near-field simulation done by $\mathrm{Xiao}^{9}$.

In the simulation a source beam with an initially Gaussian amplitude profile is created $^{29}$.

$$
E(x, y)=\exp \left[-\frac{(x \Delta x)^{2}+(y \Delta y)^{2}}{w_{0}^{2}}\right]
$$

The beam is then multiplied by the exponential of the phase screen, $\mathrm{e}^{j \theta(x, y)}$, and propagated into the far-field using a Fourier transform propagator ${ }^{31}$. The average intensity pattern in the receiver plane for a single turbulence screen can be shown in the following equation.

$$
I(x, y)=\frac{1}{S} \sum_{i=0}^{S-1} \mathscr{F}\left\{E(x, y) \exp \left[j \theta_{i}(x, y)\right] \exp [j \mathscr{T}]\right\}
$$

In (2.18), $S$ is the number of different phase screens used to construct the partially coherent beam, $\mathscr{F}$ is a two dimensional Fourier transform operator, and $\mathscr{T}$ is the 
turbulence phase screen. The Kolmogorov turbulence phase screen used was built in Matlab using the Adaptive Optics Toolbox ${ }^{30}$, where the size of the screen is equal to the size of $I(x, y)$, and the $r_{0}$ value(Fried parameter) is equal to $0.325 \mathrm{~mm}$ for the single turbulence phase screen run. Expanding this model to account for multiple turbulence screens yields.

$$
I_{k}(x, y)=\frac{1}{S} \sum_{i=0}^{S-1} \mathscr{F}\left\{E(x, y) \exp \left[j \theta_{i}(x, y)\right] \exp \left[j \mathscr{T}_{k}\right]\right\}
$$

Now $I_{k}(x, y)$ is an array of matrices where the index number $k$ refers to a different turbulence screen every $S$ frames. To scale the $x$ and $y$ axes in the observation plane a lens with a focal length of $500 \mathrm{~mm}$ was used. This scaling factor can be defined in the $x$ and $y$ directions as

$$
\Delta x_{i m g}=\frac{\lambda f_{l}}{N \Delta x} \quad \Delta y_{i m g}=\frac{\lambda f_{l}}{M \Delta y}
$$

with $\lambda$ and $f_{l}$ being equal to wavelength and focal length respectively. In Figure 2.1 an overview of the simulation setup can be seen. The parameters chosen for inputs

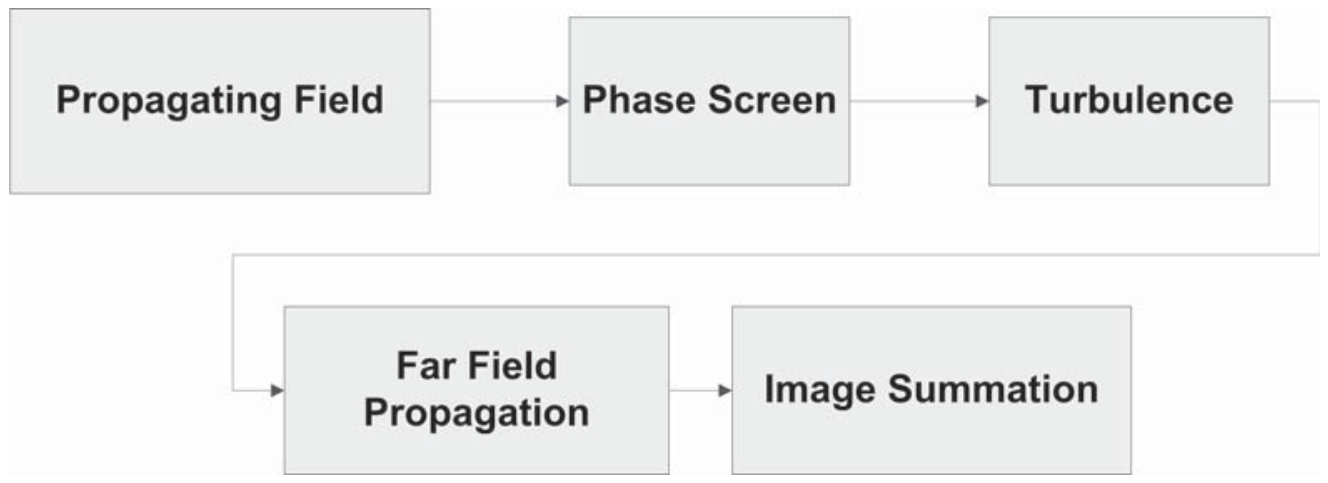

Figure 2.1: Simulation Setup

to the simulation were $\lambda=632.8 \mathrm{~nm}, w_{0}=1.7 \mathrm{~mm}, \sigma_{g}^{2}=5 * 10^{-9} \mathrm{~m}^{2}$, and $\sigma_{f}^{2}=10^{-3}$ 
$\mathrm{m}^{2}$. These parameters were chosen to satisfy the simulation requirement that ${ }^{9}$

$$
\frac{\sigma_{r}^{2}}{4 \pi \sigma_{f}^{2}} \gg 1
$$

In Figure $2.2 C(x)$ is the theoretical prediction of the infinite time average of the intensity pattern, and is provided to compare the results obtained by using a finite number of phase screens for the cross-section of $I(x, y)$. The equation for this prediction was developed by taking the following expression for optical intensity (irradiance) of a Gaussian Schell model beam ${ }^{18}$

$$
c(x, z)=\frac{1}{(\Delta(z))^{2}} \exp \left[-\frac{2(x \Delta x-(M / 2+1) \Delta x)^{2}}{w_{0}^{2}(\Delta(z))^{2}}\right]
$$

where

$$
\Delta(z)=\exp \left[1+\left(\frac{2 z}{k w_{0}^{2}}\right)^{2}\left(1+\frac{w_{0}^{2}}{\sigma_{g}^{2}}\right)\right]^{1 / 2}
$$

and transforming it into the far-field through Fourier transforms ${ }^{6,26}$, where $z$ then becomes $f_{l}$ and in this situation the resulting function $c(x, z)$ is plotted as $C(x)$ in Figure 2.2.

These results show that in the limit, as $S \rightarrow \infty$, a Gaussian intensity distribution is obtained. A summation of 30 frames was chosen for this experiment because it starts to bring in the Gaussian form and it places a lower bound on the speed of a device capable of implementing this concept in a real system.

Now that the partially coherent beam is fully defined, the next logical step is to compare the difference between using a partially spatial coherent beam and using a fully coherent beam when propagating through a turbulent atmosphere. Figure 2.3 


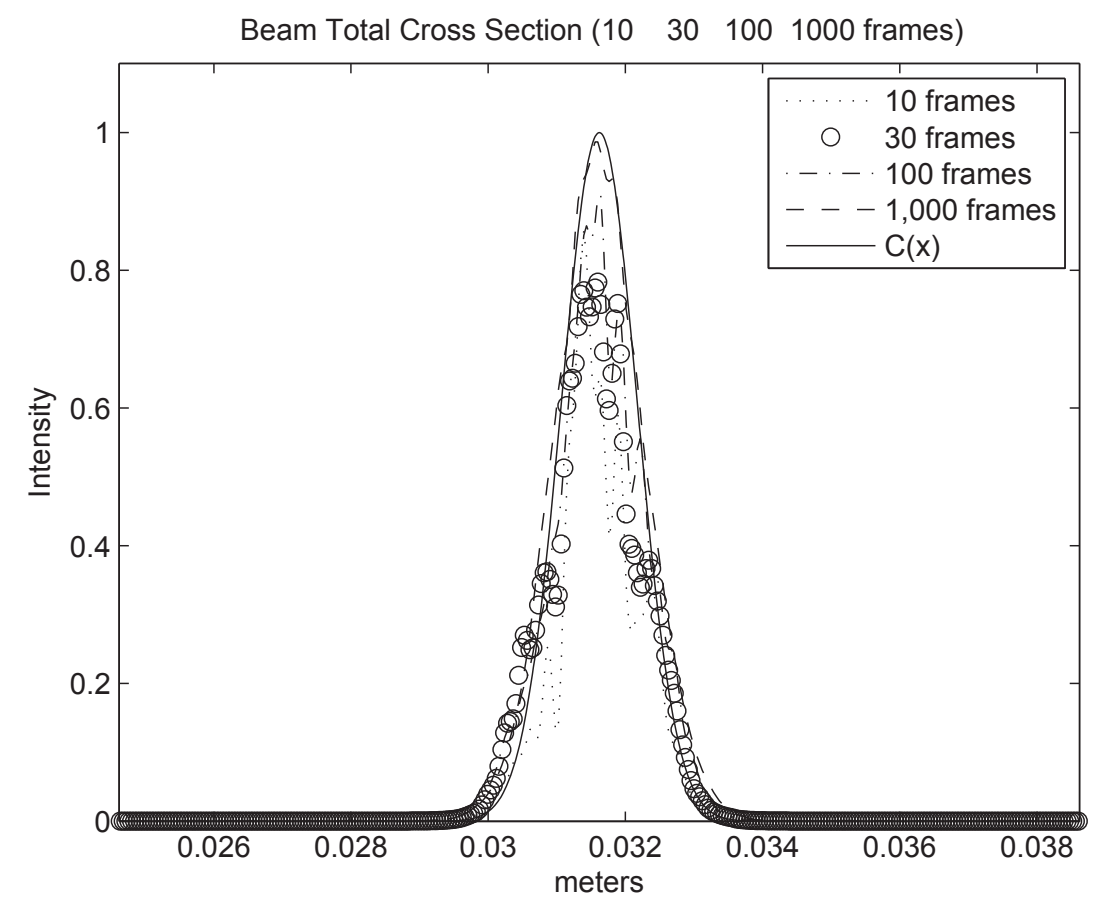

Figure 2.2: Cross sections of beam total as imaged in the far-field

shows a normalized time history of $I_{k}(x, y)$ at the center pixel. Using this figure to observe the intensity fluctuations at the center pixel through different turbulence screens shows that in an ideal case the variance of the intensity will decrease with the use of a partially spatial coherent beam. For reference the intensity of a FCS without turbulence was plotted to help illustrate the effects speckle causes.

Expanding this model to include different values of the Fried parameter, $r_{0}$, for the turbulence screen it is possible to observe the general trend of the standard deviation and mean both with and without correction in Figure 2.4. As can be seen in this figure both the standard deviation and mean intensity at the center pixel have decreased. This means the beam is more stable, but at the cost of the average intensity.

The scintillation index is a useful metric when analyzing a signal that has been 


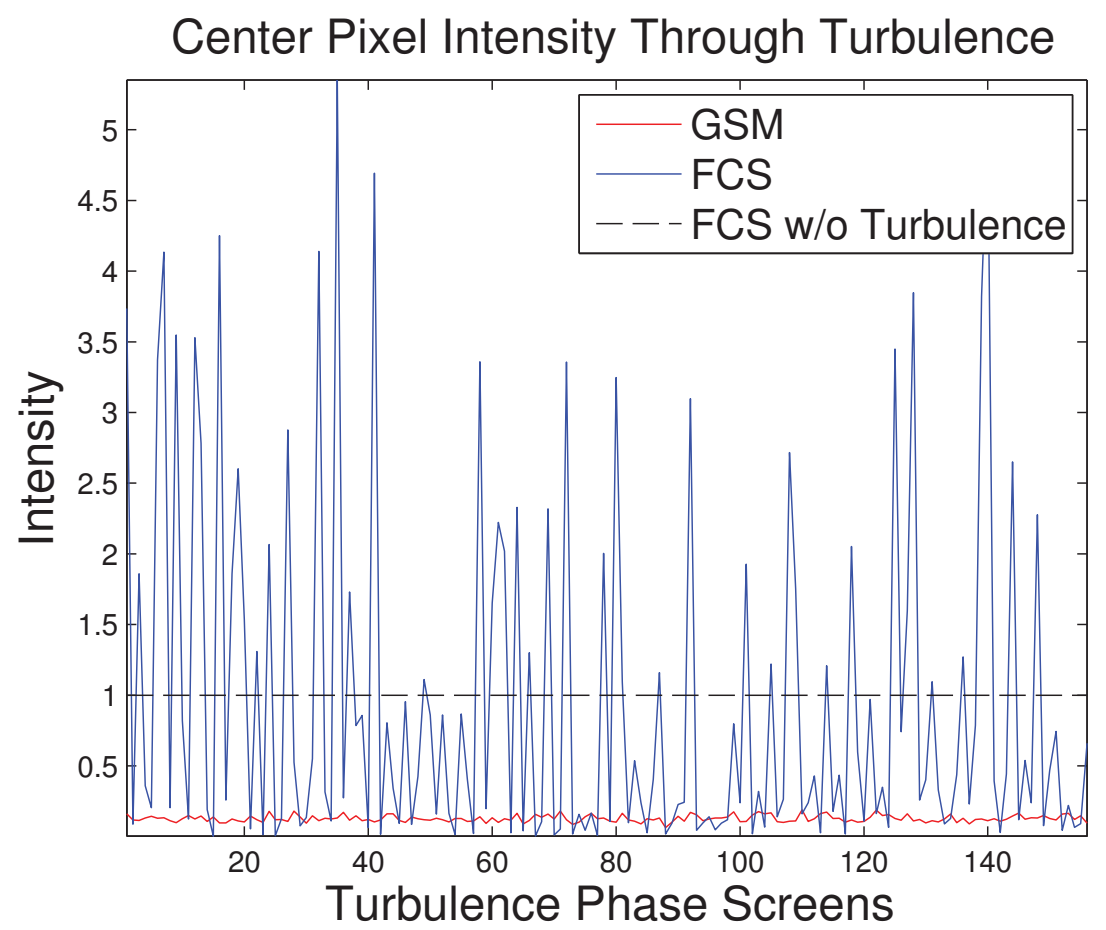

Figure 2.3: Center pixel intensity comparison through turbulence.

received after propagation through the atmosphere. The scintillation index is the variance of the received signal normalized by the mean signal. Expanding on (2.8), the equation for the scintillation index at the center pixel is

$$
S I\left(\frac{M}{2}, \frac{N}{2}\right)=\frac{\operatorname{variance}\left(I_{k}\left(\frac{M}{2}, \frac{N}{2}\right)\right)}{\left[\operatorname{mean}\left(I_{k}\left(\frac{M}{2}, \frac{N}{2}\right)\right)\right]^{2}} .
$$

In Figure 2.5 shows the scintillation index of the data in Figure 2.4. As expected the scintillation index of the partially spatial coherent beam is smaller than that of the uncorrected beam. This lower scintillation index results in decreased intensity fluctuations at the receiver. 


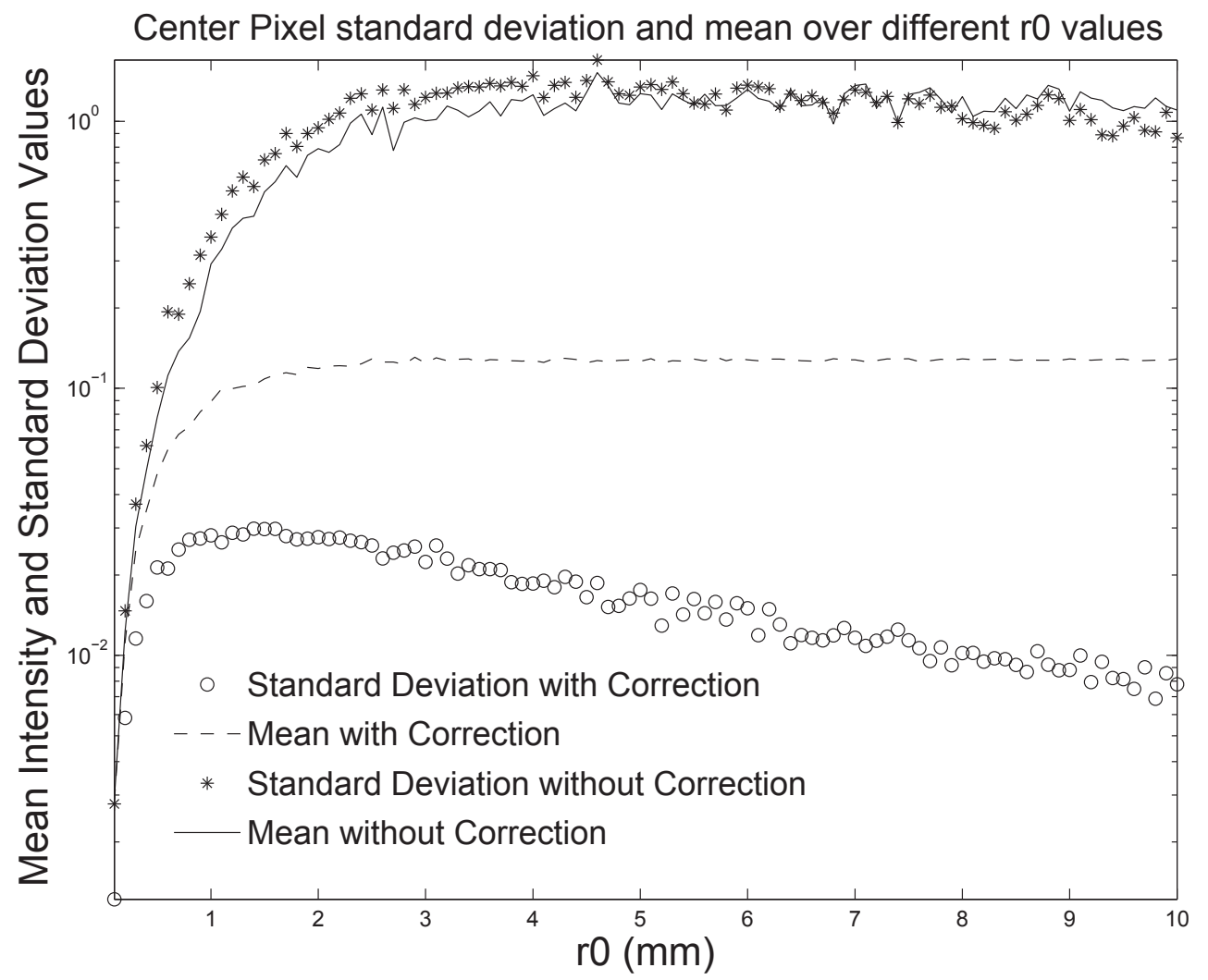

Figure 2.4: Center pixel standard deviation and mean over 156 different turbulence screens.

\subsection{Experiment}

\subsubsection{Setup}

As shown in Figure 2.6 the experiment is composed of a $632 \mathrm{~nm}$ HeNe laser that propagates through a pellicle beam splitter which then reflects from the SLM and back through the beam splitter. The beam then propagates through the Kolmogorov phase screen, Fourier transforming lens, and finally to the camera. The Fourier transform lens of focal length $500 \mathrm{~mm}$ has been placed to propagate the beam into the far-field ${ }^{31}$. Unlike previous work ${ }^{10}$, this experiment uses a rotating transmissive 


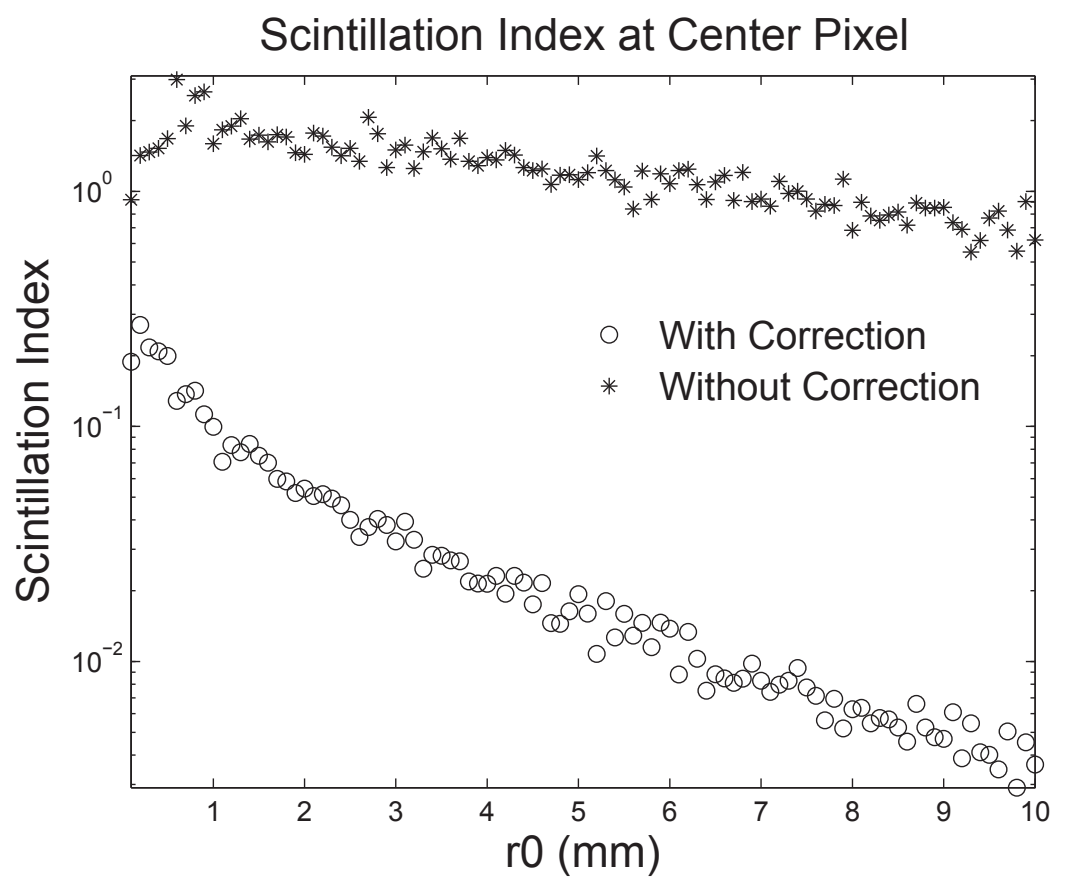

Figure 2.5: Scintillation Index over 156 different turbulence screens for each Fried parameter.

Kolmogorov phase screen, $\mathscr{T}$, built by Lexitek, Inc. ${ }^{32}$ The rotation allows new screens to be introduced without changing the turbulence strength. The turbulence strength of this screen is determined by the beam diameter that that is incident upon the screen. This is controlled through the use of two lenses with equal focal lengths, one on each side of the screen, that focus and expand the beam. The beam diameter is then dependent upon where the Kolmogorov screen is located between the lenses.

Turbulence strength in this experiment is calculated by the number of "speckles", $\mathscr{S}$, that are illuminated by a normal coherent source on the turbulence screen and is defined by

$$
\mathscr{S}=\pi\left(\frac{B_{d}\left(f_{l}-d_{s}\right)}{2 r_{0} f_{l}}\right)^{2}
$$

Where $B_{d}$ is the beam diameter of the original beam before the SLM, $f_{l}$ is the fo- 


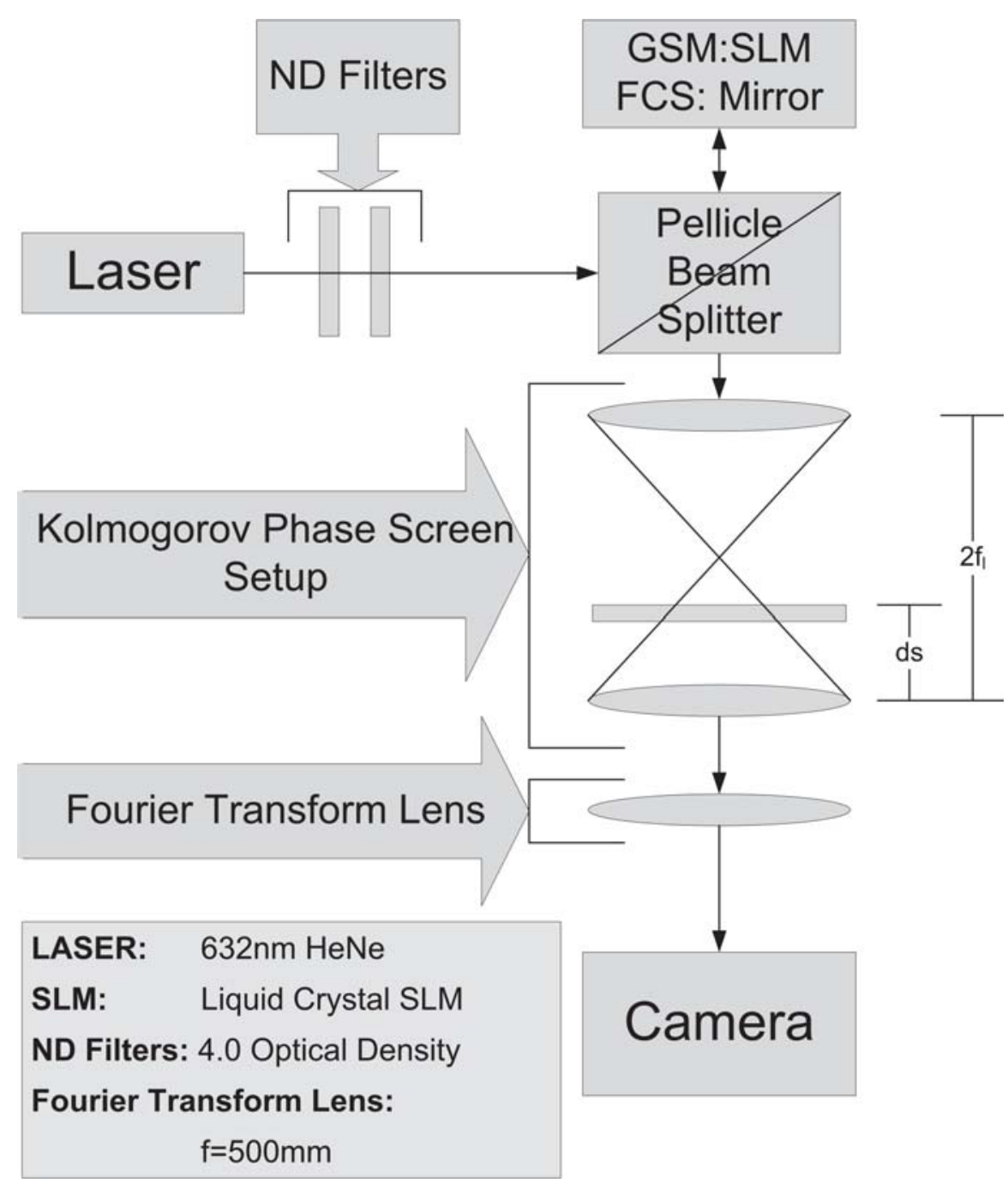

Figure 2.6: Optical Bench Setup

cal length of the controlling lenses, $d_{s}$ is the distance from the Kolmogorov phase screen to the collimating lens, and $r_{0}$ is the Fried parameter which is $0.325 \mathrm{~mm}$ for this particular screen. The value of $B_{d}$ for this experiment was approximately 3.9 $\mathrm{mm}$.

To match the simulation, partially spatial coherent beam parameters were chosen to be $\sigma_{g}^{2}=5 \times 10^{-9}$ and $\sigma_{f}^{2}=10^{-3}$. The camera had the following parameters: shutter 
speed of 30ms, collected at 15 frames per second, and no additional gain. The resolution of the camera was binned down from $1384 \times 1036$ to $640 \times 480$ for an increased frame rate, and pixel averaging. To also match the simulation, data was collected for 156 different Kolmogorov phase screens summed at 30 frames per screen; giving 4,680 total frames measured.

\subsubsection{Results}

The effects of turbulence on the intensity pattern at the receiver both with SLM correction and without can be seen in Figures 2.7-2.13. This shows experimental results of $I(x, y)$ with increasing turbulence strengths. The weakest turbulence is in Figure 2.7 with $\mathscr{S}=8.35$, and the strongest is in Figure 2.13 with $\mathscr{S}=37.2$. As expected the results in Figures 2.7-2.13 show that as turbulence strength increases the beam becomes more unstable, and the time averaged intensity pattern is more uniform and less speckled with SLM correction than without it. This observation is better illustrated in Figures 2.14-2.20, where the the radial average of Figures 2.72.13 were plotted.

The next set of experimental results in Table 2.1 are of $I(x, y)$ at specific $(x, y)$ coordinates arbitrarily chosen for being near the center of the imaged beam, and show that when you apply the partially spatial coherent beam to different turbulence strengths the variance of the pixel intensity and the scintillation index will decrease. Specifically as shown in line 2 of Table 2.1, it is possible to decrease the variance by $43 \%$ and the SI by $51 \%$ when using a partially spatial coherent beam in this setup.

Figure 2.21 shows the calculated scintillation index reduction of the experimental $I(x, y)$ at 200 different arbitrarily chosen $(x, y)$ coordinates near the center of the 

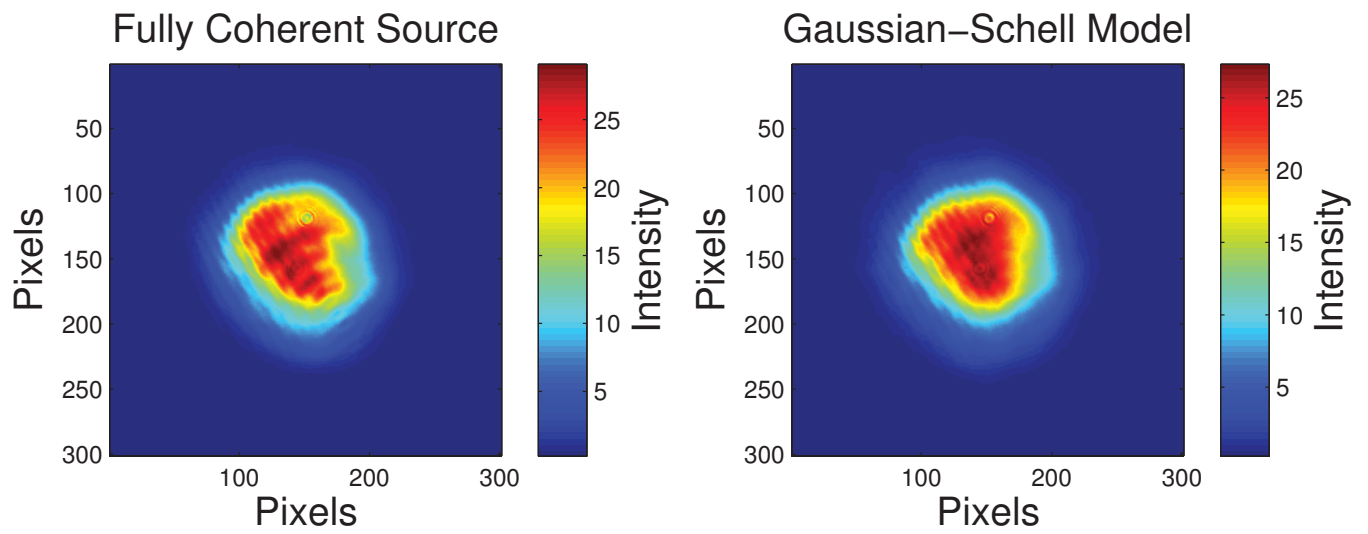

Figure 2.7: Beam comparison of a partially spatial coherent beam to a coherent LASER source. The maximum values in the transmitted beam are red, and the minimum values are dark blue. Turbulence strength of $\mathscr{S}=8.35$.

Table 2.1

Variance analysis of received intensity data

\begin{tabular}{|l|l|l|l|l|l|l|l|l|}
\hline & \multicolumn{2}{|c|}{ With SLM } & \multicolumn{2}{c|}{ Without SLM } & Variance & \multicolumn{3}{c|}{$S I$} \\
\hline $\mathscr{S}$ & Variance & Mean & Variance & Mean & Decrease & $S I_{w}$ & $S I_{w o}$ & Decrease \\
\hline 15.6 & 5.45 & 21.5 & 10.6 & 22.3 & $49 \%$ & 0.012 & 0.021 & $45 \%$ \\
25.3 & 13.0 & 18.7 & 23.0 & 17.4 & $43 \%$ & 0.037 & 0.076 & $51 \%$ \\
30.9 & 13.9 & 17.3 & 19.4 & 16.4 & $28 \%$ & 0.046 & 0.072 & $36 \%$ \\
\hline
\end{tabular}

receiver for each turbulence strength. The reason for the gaps in percent reduction in this graph are caused by the arbitrary choosing of the $(x, y)$ coordinates. By using a histogram to analyze this data, it can be seen in Figure 2.22 that the majority of the scintillation index reduction near the center occurs in the range of $30-50 \%$.

By expanding the area of analysis to include the entire captured data set, it is easily observable in Figure 2.23 that for the majority of the receivers the calculated SI is reduced using the GSM. Figure 2.24 shows a histogram of the data in Figure 2.23. The overall calculated mean SI reduction for all measured turbulence strengths is $19.70 \%$, with a standard deviation of 12.97 . These results show that regardless of turbulence strength the GSM of partial coherence is an effective means for reducing 

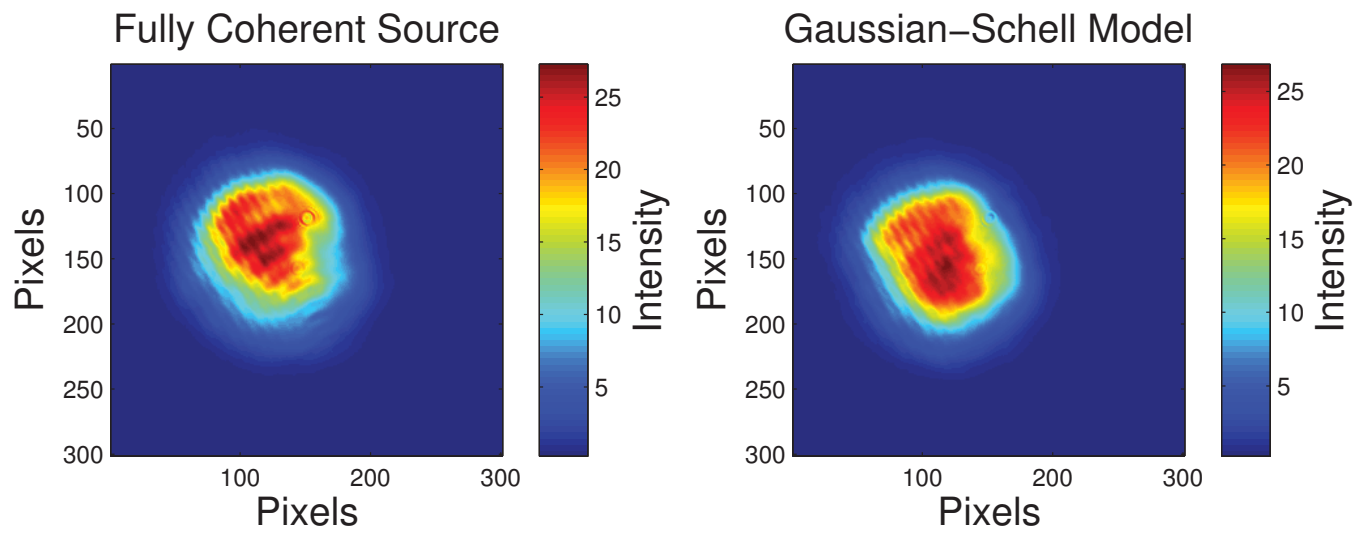

Figure 2.8: Beam comparison of a partially spatial coherent beam to a coherent LASER source. The maximum values in the transmitted beam are red, and the minimum values are dark blue. Turbulence strength of $\mathscr{S}=11.7$.

the intensity fluctuations of the received field when compared to a highly coherent laser source.

\subsection{Conclusion and Future Work}

This chapter has shown that using a liquid crystal SLM is a practical proof of concept device when implementing a partially spatial coherent beam in an experiment. Keep in mind that the frame rate of liquid crystal technology needs to develop several orders of magnitude more before it is truly appropriate for high bandwidth FSO communications. With the current pace of technology it is entirely possible that another SLM technology will emerge to accomplish this in the future. This chapter has also shown, through the scintillation index, that when using a partially spatial coherent beam in the far-field, the variance of the pixel intensity will be less than that of a fully coherent source in the same turbulence. This lower variance was achieved not through the use of expensive adaptive optics, but instead by applying statistical phase variations to the propagating field at the source. This demonstrates 

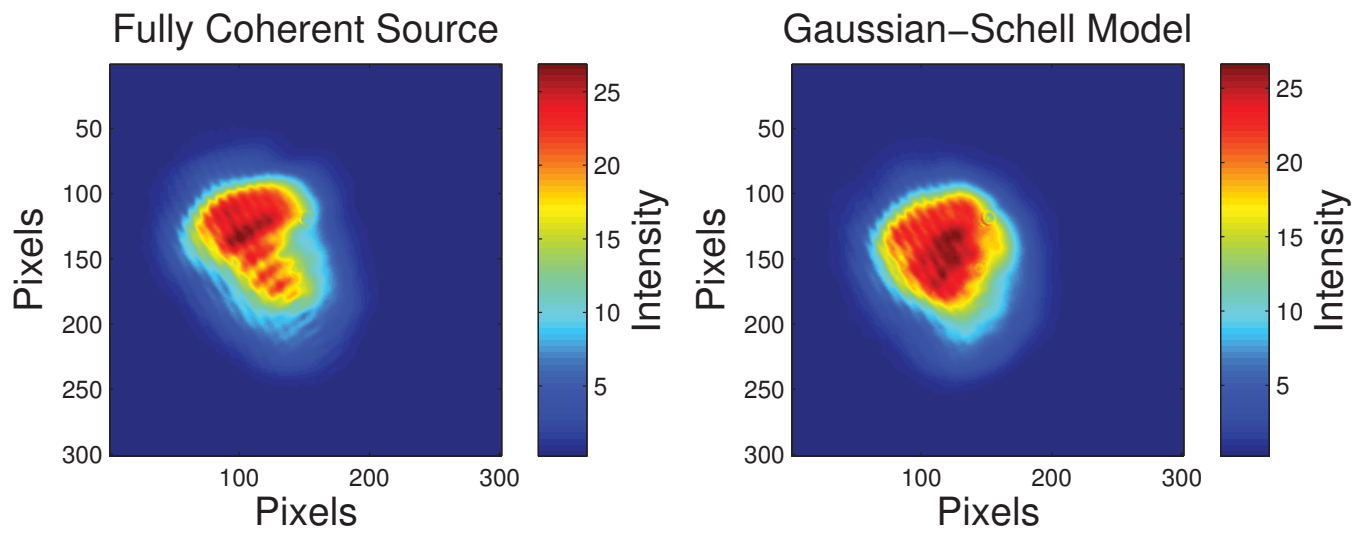

Figure 2.9: Beam comparison of a partially spatial coherent beam to a coherent LASER source. The maximum values in the transmitted beam are red, and the minimum values are dark blue. Turbulence strength of $\mathscr{S}=15.7$.

that partial coherence created with a high resolution spatial light modulator is an effective method for reducing the effects of scintillation due to atmospheric turbulence, instead of rotating phase diffusers, multi mode lasers, and traditional adaptive optics. In the end it has been shown both theoretical and experimentally that with the given parameters partial coherence will ultimately decrease the scintillation index, thereby increasing the signal to noise ratio in a FSO communications channel.

Future work on the partially spatial coherent beam to be accomplished is developing a quasi-random model that more accurately and precisely predicts what phase variations are needed to be applied to the propagating field. This would lead to fewer individual phase screens per turbulence path to create the correct beam profile. Fewer summations would also lead to a more effective optical communications channel.

Another aspect that should be considered is an experiment to add a non-Kolmogorov turbulence path, in the form of another SLM to simulate this path, or by using an 

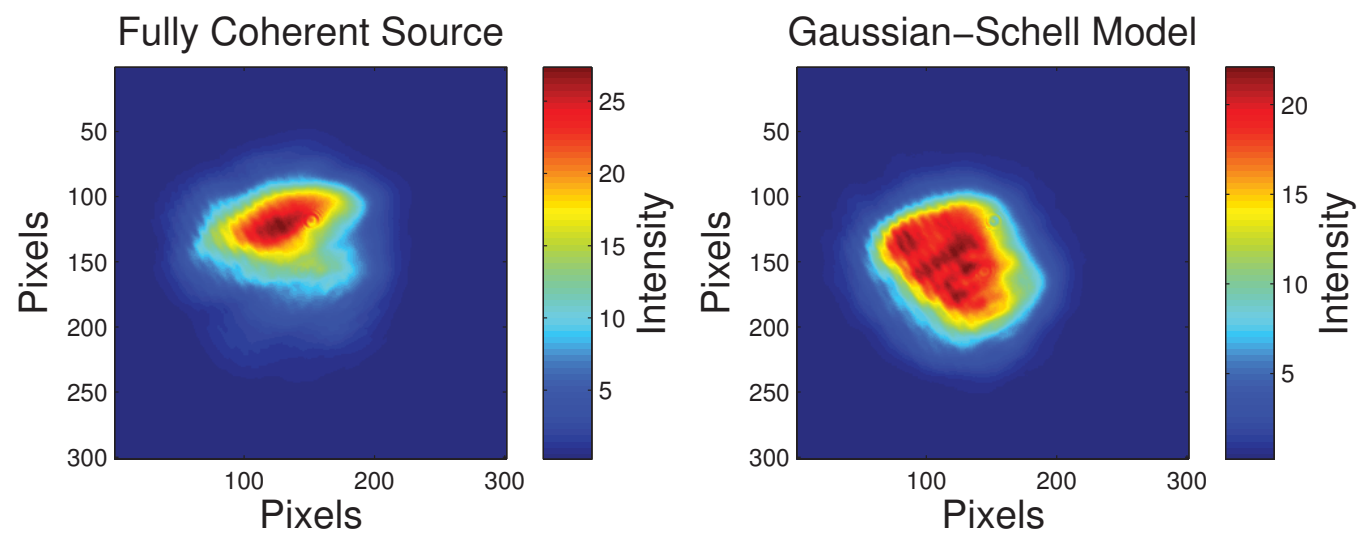

Figure 2.10: Beam comparison of a partially spatial coherent beam to a coherent LASER source. The maximum values in the transmitted beam are red, and the minimum values are dark blue. Turbulence strength of $\mathscr{S}=20.2$.

existing free space optical channel. These experiments will help to model how the partially coherent beam behaves when introduced to other turbulence types in a free space optical channel. 

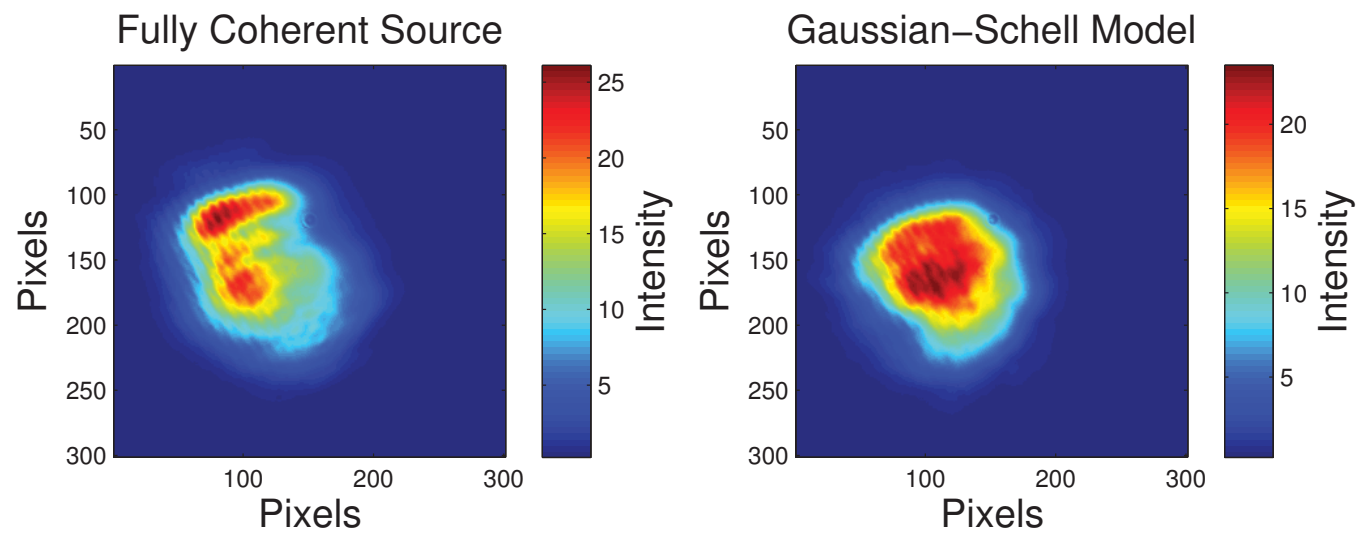

Figure 2.11: Beam comparison of a partially spatial coherent beam to a coherent LASER source. The maximum values in the transmitted beam are red, and the minimum values are dark blue. Turbulence strength of $\mathscr{S}=25.3$.
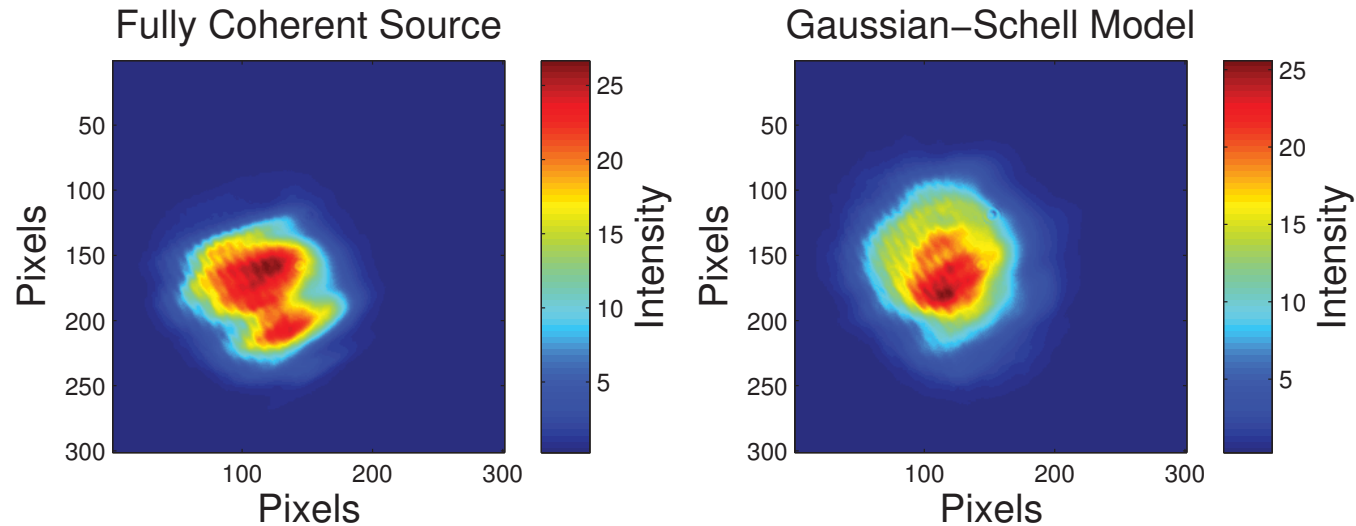

Figure 2.12: Beam comparison of a partially spatial coherent beam to a coherent LASER source. The maximum values in the transmitted beam are red, and the minimum values are dark blue. Turbulence strength of $\mathscr{S}=31.0$. 

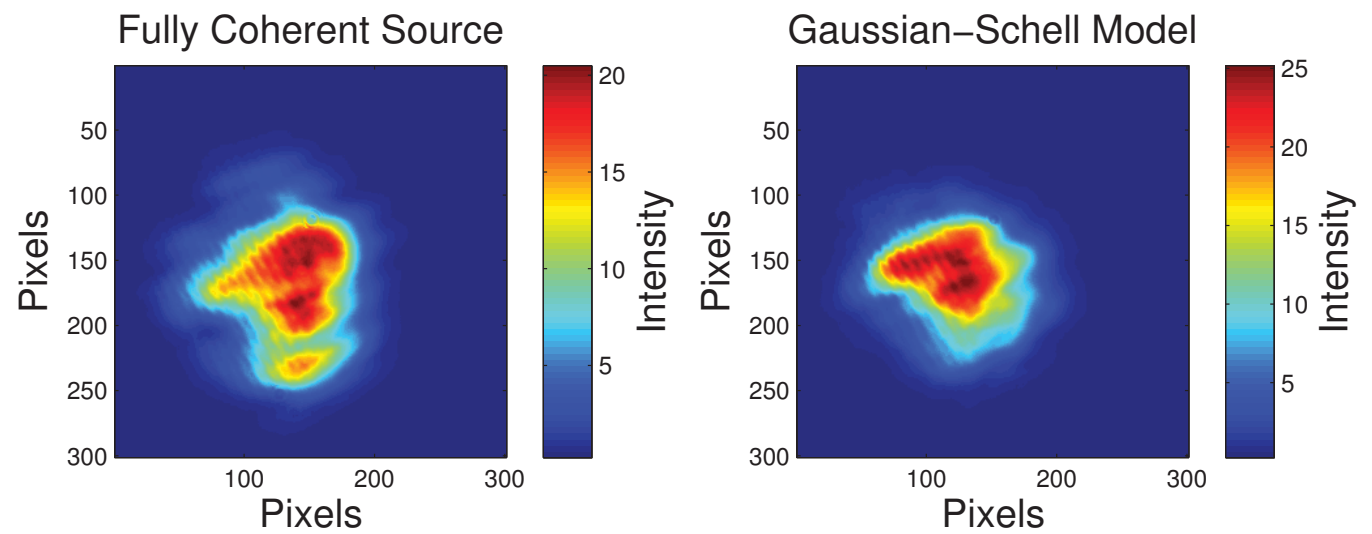

Figure 2.13: Beam comparison of a partially spatial coherent beam to a coherent LASER source. The maximum values in the transmitted beam are red, and the minimum values are dark blue. Turbulence strength of $\mathscr{S}=37.2$.
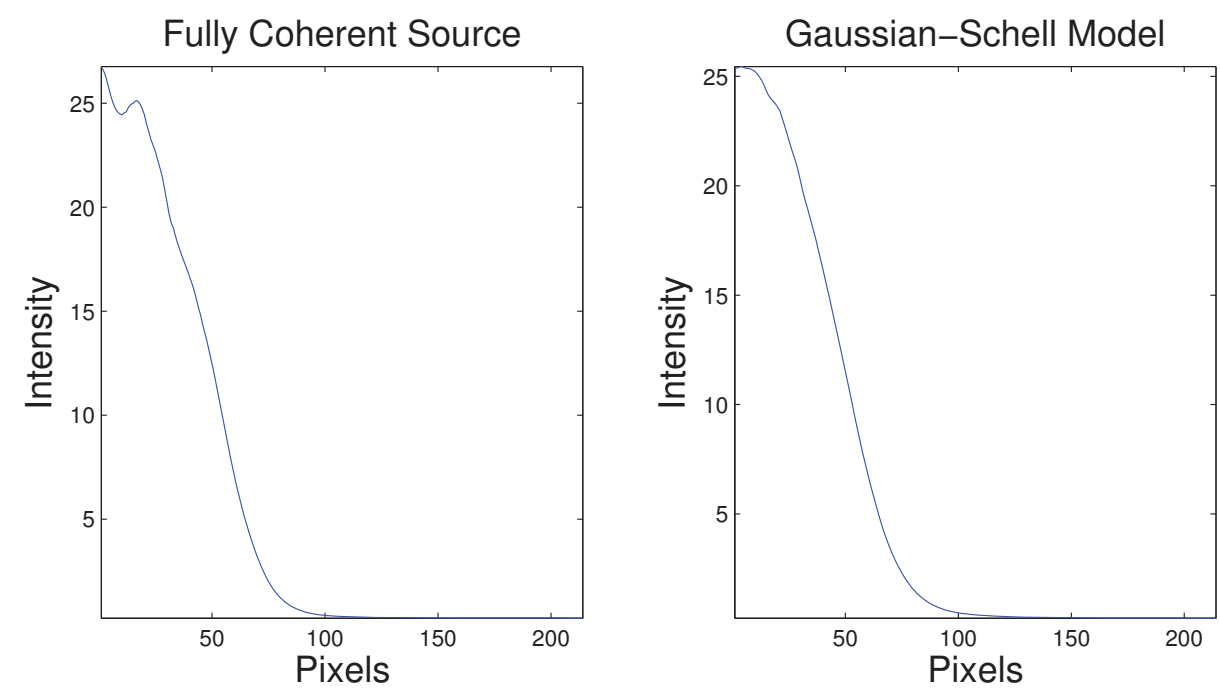

Figure 2.14: Radial average beam comparison of a partially spatial coherent beam to a coherent LASER source. Turbulence strength of $\mathscr{S}=8.35$. 

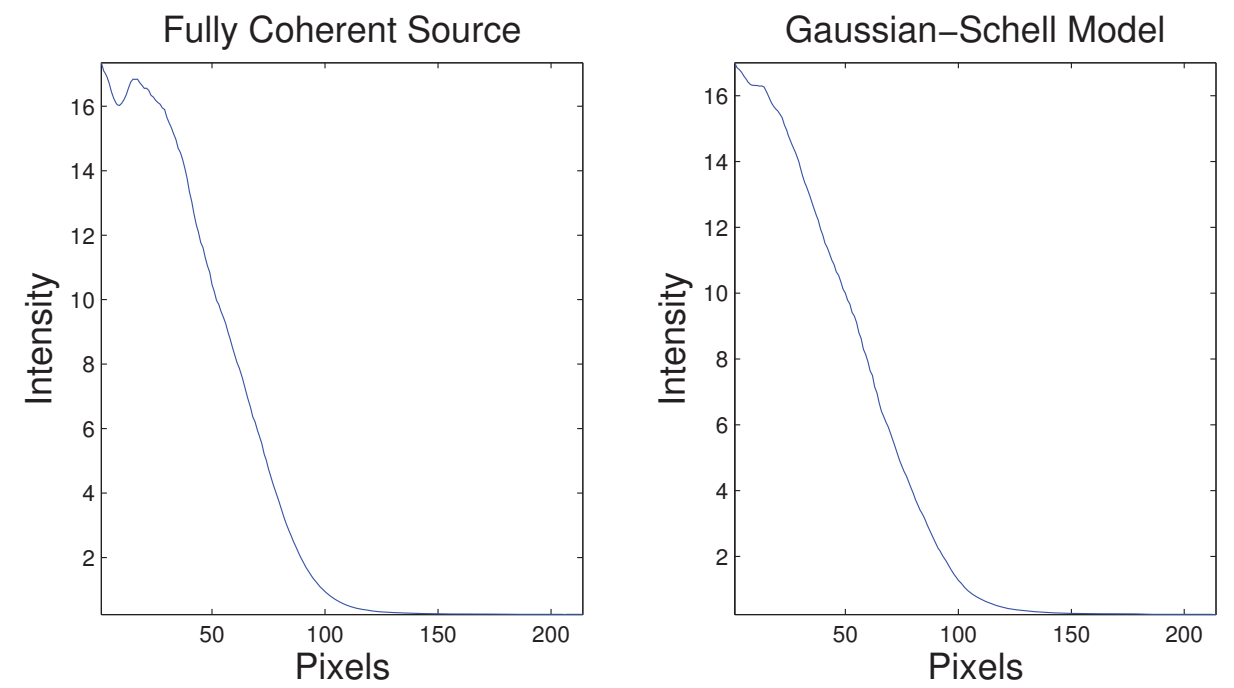

Figure 2.15: Radial average beam comparison of a partially spatial coherent beam to a coherent LASER source. Turbulence strength of $\mathscr{S}=11.7$.
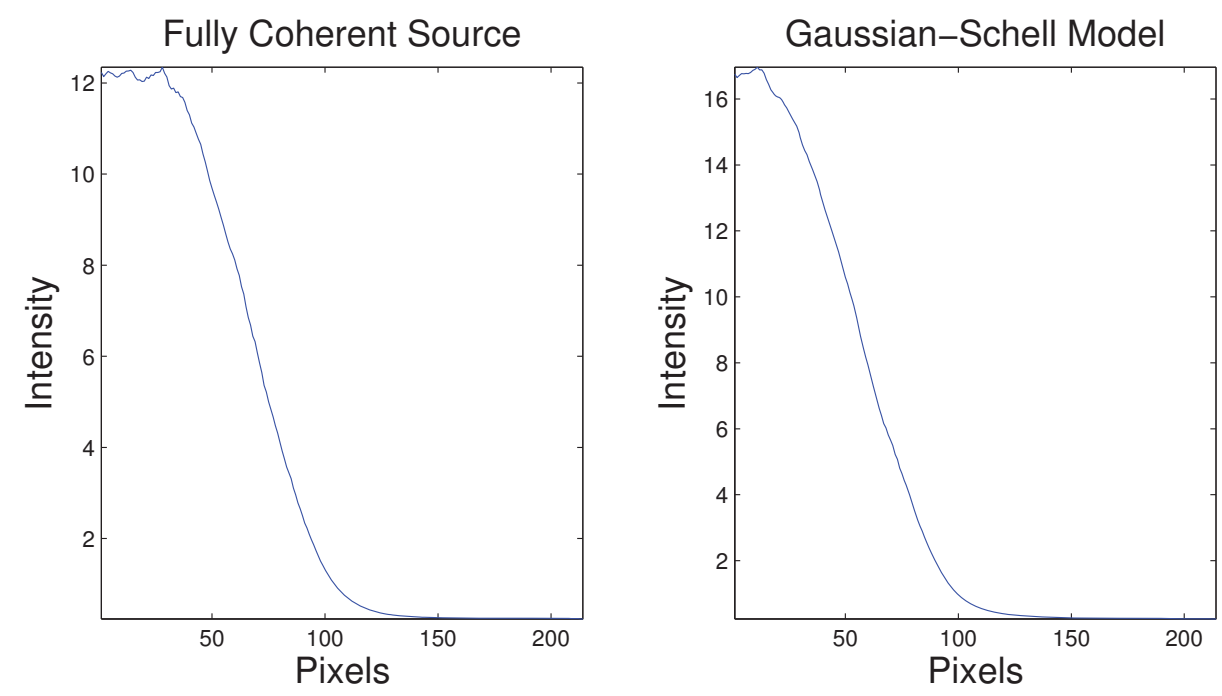

Figure 2.16: Radial average beam comparison of a partially spatial coherent beam to a coherent LASER source. Turbulence strength of $\mathscr{S}=15.7$. 

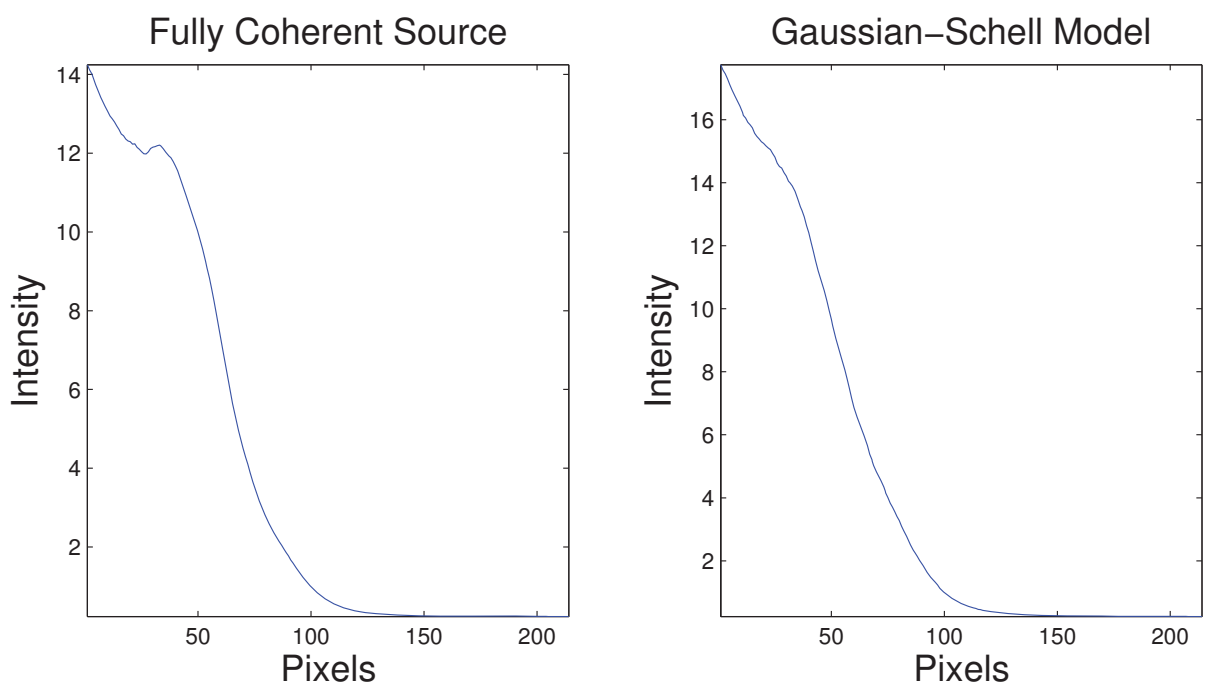

Figure 2.17: Radial average beam comparison of a partially spatial coherent beam to a coherent LASER source. Turbulence strength of $\mathscr{S}=20.2$.
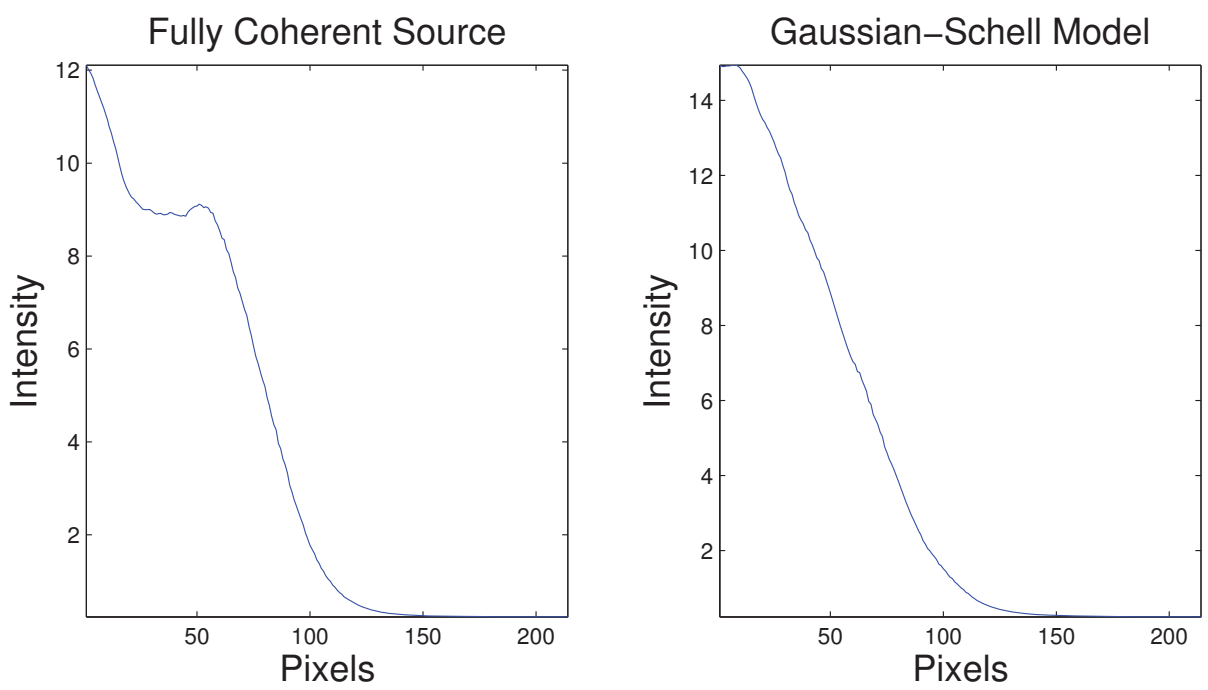

Figure 2.18: Radial average beam comparison of a partially spatial coherent beam to a coherent LASER source. Turbulence strength of $\mathscr{S}=25.3$. 

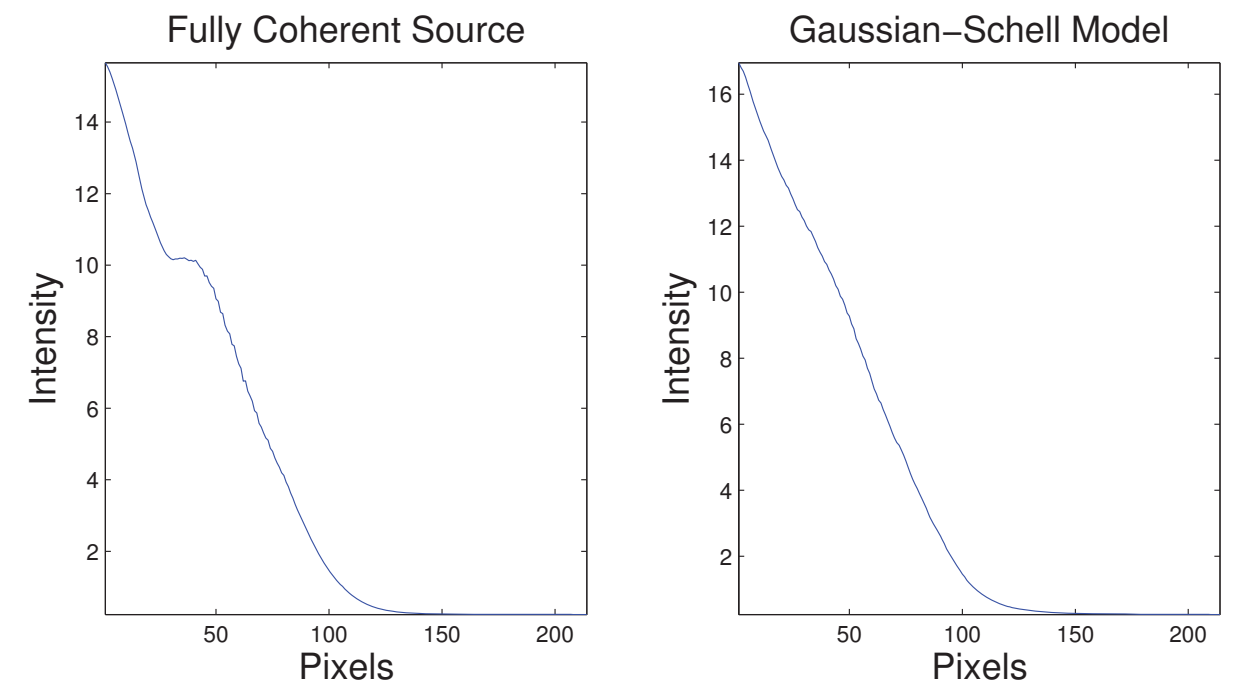

Figure 2.19: Radial average beam comparison of a partially spatial coherent beam to a coherent LASER source. Turbulence strength of $\mathscr{S}=31.0$.
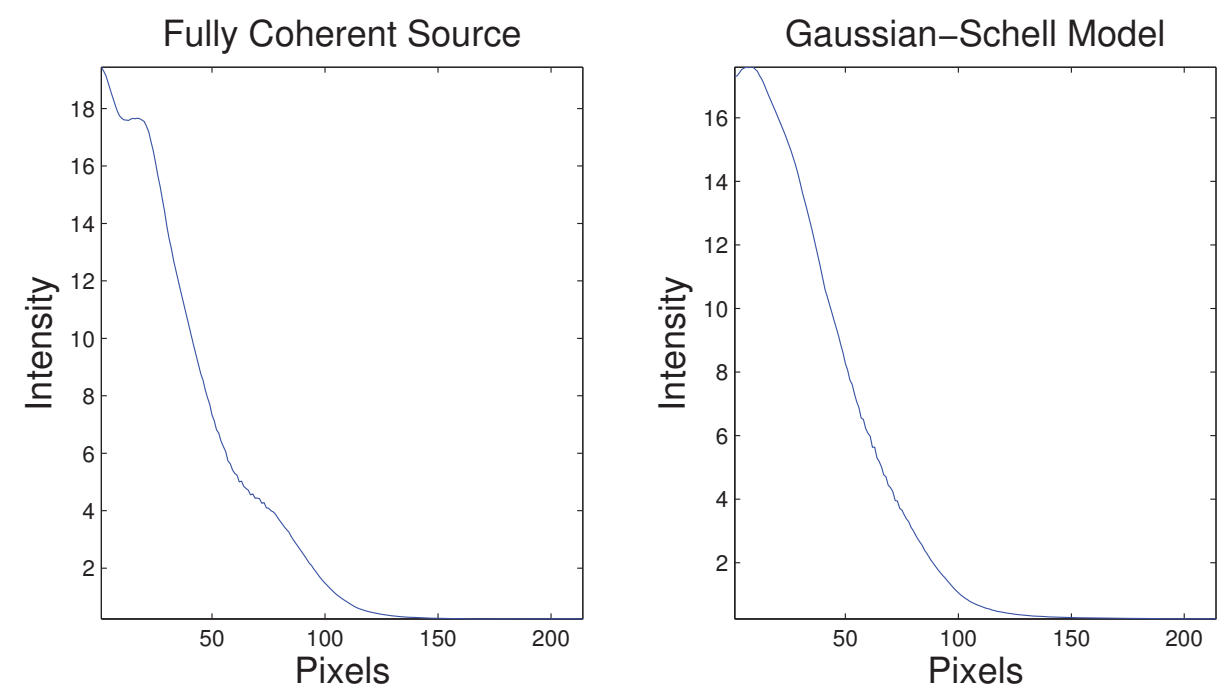

Figure 2.20: Radial average beam comparison of a partially spatial coherent beam to a coherent LASER source. Turbulence strength of $\mathscr{S}=37.2$. 


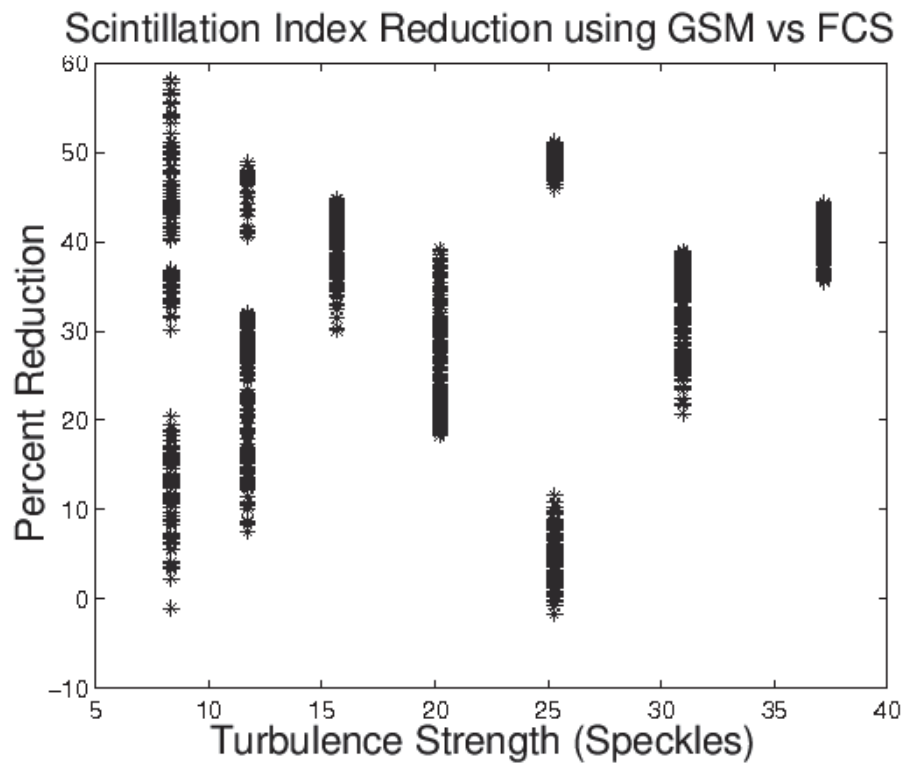

Figure 2.21: Calculated scintillation index reduction data for $200(x, y)$ coordinates near the center of the receiver for each turbulence strength.

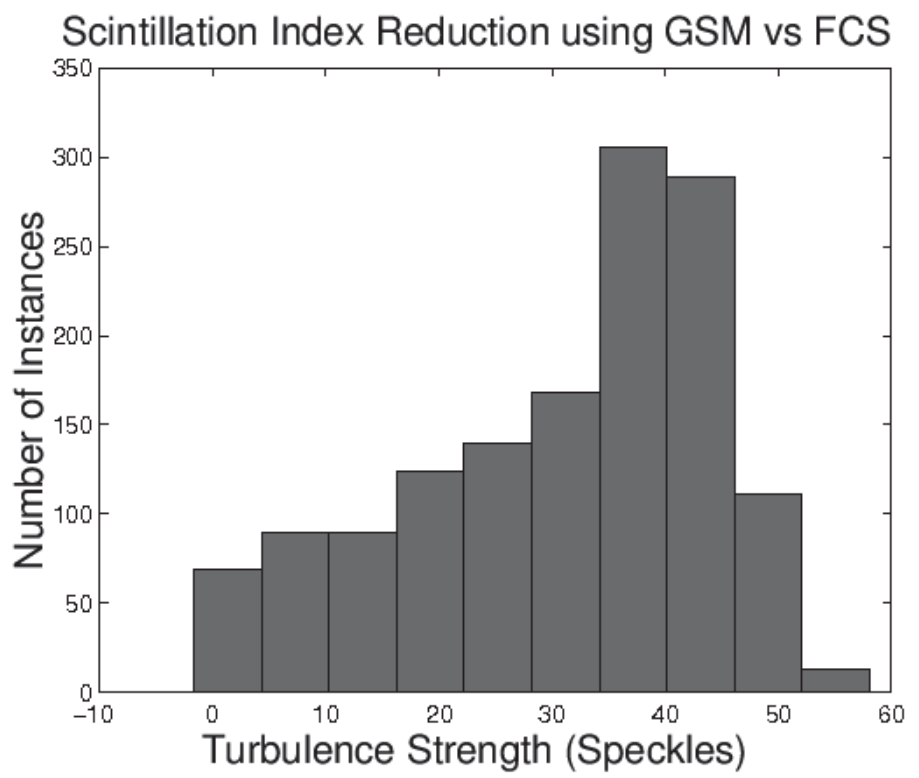

Figure 2.22: Histogram of the scintillation index reduction data in Figure 2.21. 


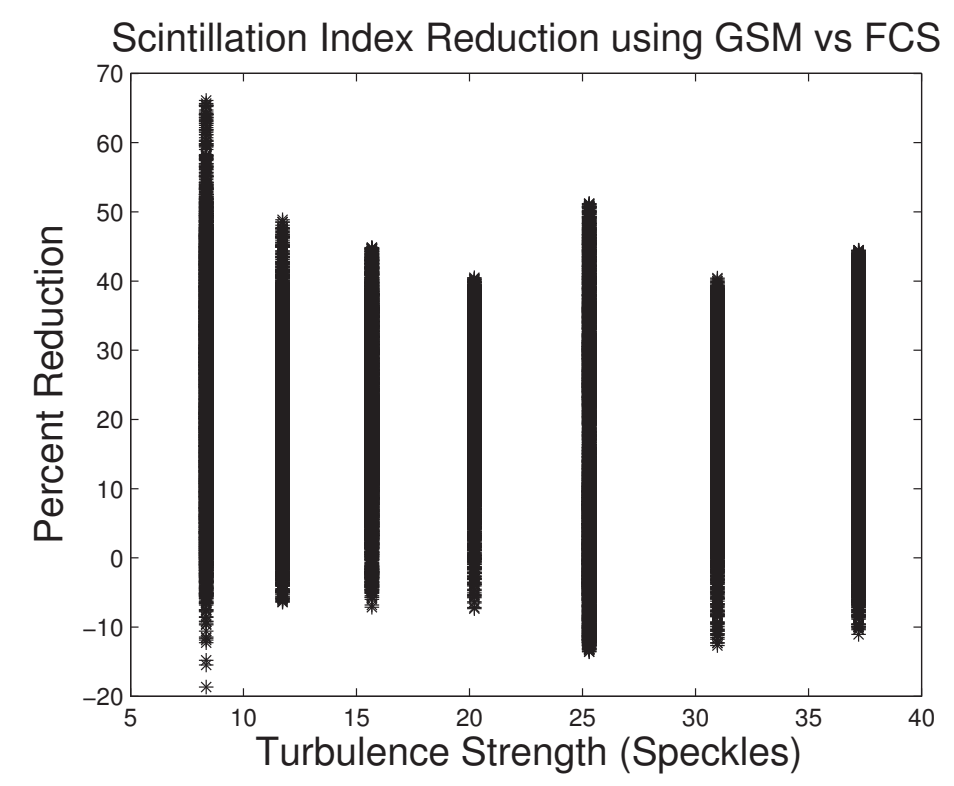

Figure 2.23: Spread of the calculated scintillation index reduction over the entire receiver for each turbulence strength. There are 307,200 calculated values for each turbulence strength.

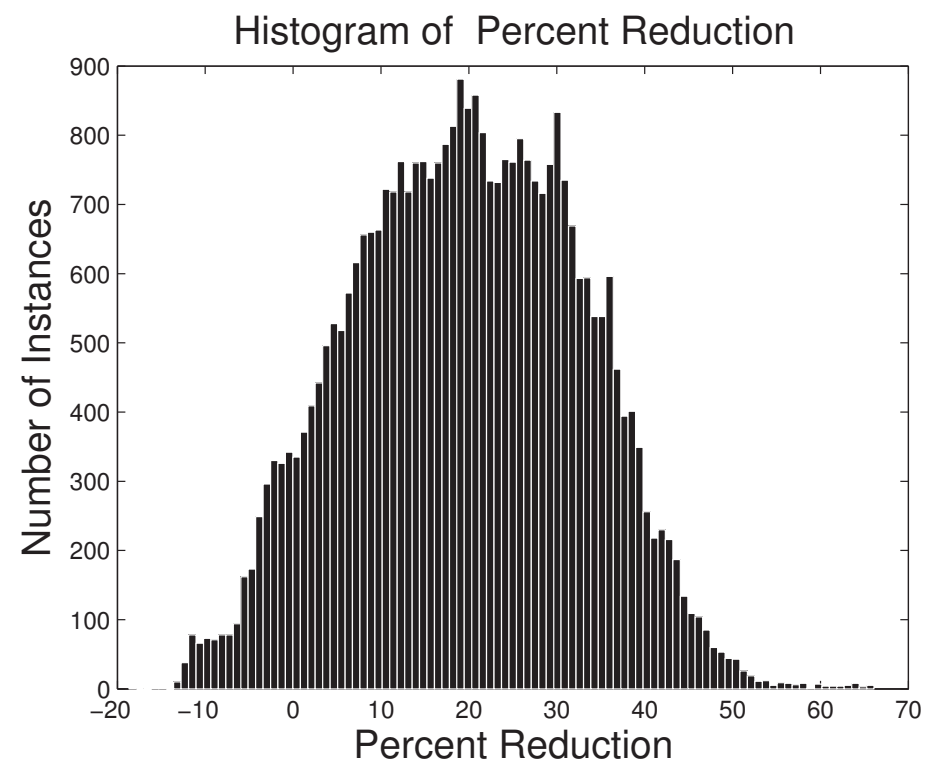

Figure 2.24: Histogram of the scintillation index reduction data in Figure 2.23. 


\section{Chapter 3}

\section{Upper Coherence Limit}

\subsection{Introduction}

It is well documented that turbulence affects traditional free space optical communications by creating a speckled field at the receiver. ${ }^{1-6}$ Partially coherent beams (PCB) have been shown to mitigate the effects of turbulence in free space optical(FSO) communications. ${ }^{6,8-11,13-17}$ Unfortunately most of these solutions tend to be limited to specific turbulent regimes, and require knowledge of the turbulence to adjust the beam parameters accordingly. In this chapter a method is proposed to control a type of PCB, the Gaussian-Schell model ${ }^{18,19}$ (GSM) beam, to optimize the beam parameters by limiting the spatial correlation width to mitigate turbulence effects, and to minimize the mean variance of the received power while maintaining the signal-to-noise ratio(SNR).

One of the problems with using a PCB in FSO communications is that the beam

diverges faster than a fully coherent source (FCS) in atmospheric turbulence ${ }^{8,16}$ resulting in a beam with a lower scintillation index (SI) at the cost of a reduced optical power density at the receiver. One of the goals of this work is to determine 
an optimal point in which to limit the PCB and maximize the coherence of the source, therefore limiting the reduction of the optical power density.

Due to the difficulty in developing a theoretical expression for the mean and variance of the received power in strong turbulence a wave optics simulation is used to study this issue. In this approach the spatial correlation width, $\sigma_{g}^{2}$, of a simulated GSM beam through turbulence is increased from spatially incoherent to spatially coherent, and the received irradiance is saved for statistical analysis. In this approach it is assumed that the GSM beam is controlled through the use of a Spatial Light Modulator(SLM). The statistics from the saved irradiance are used to determine the coherence properties of the GSM beam which optimize the beneficial aspects of a partially coherent transmitting beam. This value of $\sigma_{g}^{2}$ is defined as the upper-coherence limit(UCL) for the GSM beam in a turbulent optical channel. After the UCL has been determined, it is sent via a low bandwidth communication channel to the transmitter. This closed loop is essential for providing the transmitter with the correct parameters to correct for the atmospheric fluctuations. The driving force behind the creation of this limit is to determine if there is a simple process to automatically determine optimal beam parameters for a GSM beam in a closed communication system.

This chapter demonstrates a straight forward method to calculate the optimal spatial correlation width of a GSM beam when channel turbulence is unknown with a low bandwidth feedback channel completing the closed loop communication system. The selection of $\sigma_{g}^{2}$ is obtained by calculating the UCL, which this chapter will show is determined in a straightforward manner, regardless of turbulence strength or receiver aperture size.

The remainder of the chapter is organized as follows. In section 3.2 the mathematical details of the simulated GSM and fully coherent source(FCS) are discussed. 
In section 3.3 the details of the simulation: channel overview, constants, and an analysis of the method are examined. Finally in section 3.4 the conclusions are presented with future work ideas.

\subsection{Theory}

Starting with the definition of spatial power spectral density (PSD) of the index of refraction fluctuations for a horizontal turbulent channel where the spatial PSD of the index of refraction fluctuations is described by the Kolmogorov spectrum represented by ${ }^{7}$

$$
\Phi_{n}^{K}(k, z)=0.033 C_{n}^{2}(z) k^{-11 / 3},
$$

where $k$ is the scalar wavenumber, and $C_{n}^{2}$ is the structure constant of the index of refraction fluctuations with units $\mathrm{m}^{-2 / 3}$. Since a horizontal channel is being modeled, $C_{n}^{2}$ is kept constant and a single phase screen, $\mathscr{T}(x, y)$, is placed in the pupil of the system to model the atmospheric turbulence effects. The strength of these screens is controlled by the Fried parameter, $r_{0}$, with the relationship between $r_{0}$ and $C_{n}^{2}$ given by

$$
r_{0}=0.185\left[\frac{4 \pi^{2}}{k^{2} C_{n}^{2} z_{i}}\right]^{3 / 5}
$$

where $z_{i}$ is the propagation distance between turbulence layers. Since the channel is assumed horizontal, there is only one layer, therefore $z_{i}=z$, with $z$ being defined as the distance of propagation.

A single time averaged instance of the irradiance of the transmitting beam in 
the receiver plane is

$$
P C B(x, y)=\frac{1}{S} \sum_{i=1}^{S}\left|\mathscr{F}^{-1}\left\{\mathscr{F}\left\{E(x, y) \exp \left[j \theta_{i}(x, y)\right] \exp [j \mathscr{T}(x, y)]\right\} H(u, v)\right\}\right|^{2}
$$

where $H$ is the transfer function of the Fresnel propagator and is defined as ${ }^{31}$

$$
H(u, v)=\mathscr{F}\left\{\frac{\exp [j k z]}{j \lambda z} \exp \left[\frac{j k}{2 z}\left((x \Delta x)^{2}+(y \Delta y)^{2}\right)\right]\right\}
$$

where $\mathscr{F}$ is a two-dimensional Fourier transform operator, $u$ and $v$ are spatial frequencies, $\lambda$ is the wavelength, and $k$ is the scalar wave number defined by

$$
k=\frac{2 \pi}{\lambda}
$$

It should be noted that $x=(0,1, \ldots N-1)$ and $y=(0,1, \ldots M-1)$, where $N$ and $M$ are equal to the number of adjustable pixels of the simulated Spatial Light Modulator(SLM), that is controlling the GSM, in the $x$ and $y$ directions respectively. $\Delta x$ and $\Delta y$ are the spatial sample spacing in the $x$ and $y$ directions.

When using the Fresnel propagator in a simulation, it is necessary to properly address spatial sampling issues. The transfer function is said to be critically sampled when $\Delta x=\lambda z / L$, with $L$ being the physical length of array $x$ in meters. When $\Delta x<\lambda z / L$ the transfer function is undersampled. In the undersampled scenario the simulation is valid for relatively "long" propagation distances. ${ }^{33}$

$E(x, y)$ is the initial amplitude of the Gaussian source and is defined as ${ }^{29}$

$$
E(x, y)=\exp \left[-\frac{(x \Delta x)^{2}+(y \Delta y)^{2}}{w_{0}^{2}}\right]
$$


$\theta(x, y)$ is the controlling phase screen of the GSM and is defined as ${ }^{9,13}$

$$
\begin{aligned}
\theta(x, y)=\mathscr{F}^{-1}\left\{\mathscr{F}\left\{\frac{1}{2 \pi \sigma_{f}^{2}} \exp \left[-\frac{(x \Delta x)^{2}+(y \Delta y)^{2}}{2 \sigma_{f}^{2}}\right]\right\}\right. \\
\left.\mathscr{F}\left\{\frac{(\gamma(x, y)-.5) \sigma_{r}}{(\Delta x \Delta y)^{2}}\right\}\right\}(\Delta x \Delta y)
\end{aligned}
$$

where $\gamma(x, y)$ is a matrix of random draws from a random number generator whose output is uniformly distributed on $[0,1], \sigma_{r}$ relates to the amplitude variation of the phase screen, and $\sigma_{f}$ is the transverse spatial correlation length. Both $\sigma_{r}$ and $\sigma_{f}$ have units of length.

The spatial correlation width, $\sigma_{g}^{2}$, of $\theta$ is ${ }^{9,13}$

$$
\sigma_{g}^{2}=\frac{8 \pi \sigma_{f}^{4}}{\sigma_{r}^{2}}
$$

Physically $\sigma_{g}^{2}$ is a measure of the $\mathrm{e}^{-1}$ radius of the correlation function; more importantly it is a measure of the spatial coherence of the beam. It is important to remember that the larger $\sigma_{g}^{2}$ is, the more coherent the propagating beam will be. This useful fact allows us to create both a partially spatial coherent beam, as well as a fully coherent beam.

The instantaneous intensity at the receiver plane of a normal propagating coherent laser source through horizontal turbulence is defined as

$$
f c s(x, y)=\left|\mathscr{F}^{-1}\{\mathscr{F}\{E(x, y) \exp [j \mathscr{T}(x, y)]\} H(u, v)\}\right|^{2} .
$$




\subsection{Simulation}

\subsubsection{Channel Description}

This wave optics simulation is an extension of simulations used in previous efforts $^{9,13,34}$ and has been extensively tested, and compared to theory when possible. This FSO channel is primarily composed of two parts, the optical channel and the feedback channel. The feedback channel is assumed to be a simple low bandwidth radio frequency $(\mathrm{RF})$ communications channel that sends the current UCL and received power level to the transmitter to optimize the transmission parameters of the partially coherent beam. If the received power becomes too low the receiver will reset to calibration mode. The transmitter will recognize this by the low power level sent by the receiver, and sweep through a predetermined set of $\sigma_{g}^{2}$ parameters to redefine optimal settings. It is assumed that the actual analysis will occur within the receiver, keeping transfered data on the low bandwidth channel to a minimum, and ensuring that any actual communications data is not accidentally retransmitted over the feedback channel. A simple system overview can be seen in Figure 3.1.

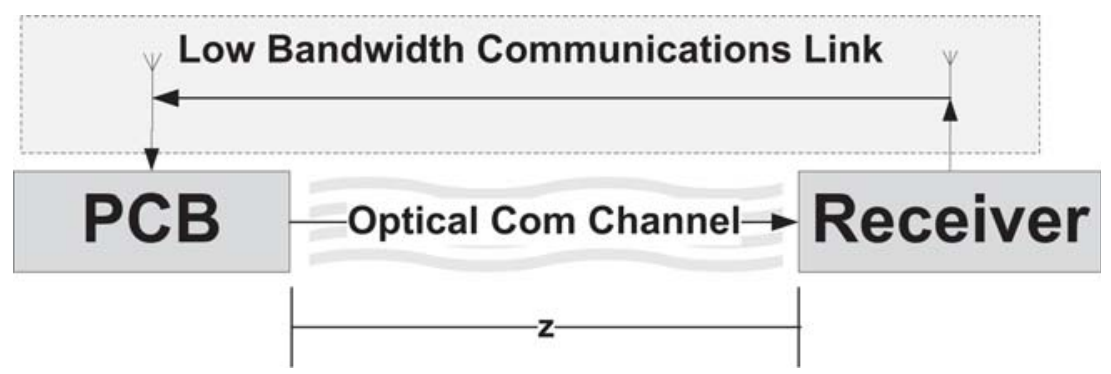

Figure 3.1: Free Space Optical Communications Channel Overview 


\subsubsection{Constants}

The simulation is broken up into two separate models, the small aperture model and the large aperture model. The small aperture model is a $10 \mathrm{~cm}$ by $10 \mathrm{~cm}$ atmospheric cross-section that is propagated $100 \mathrm{~m}$, and the large aperture model is a $3 \mathrm{~m}$ by $3 \mathrm{~m}$ atmospheric cross-section that is propagated $17,778 \mathrm{~m}$. At the receiver each crosssection is then broken down into different aperture sizes. This was done to show the

versatility of the technique, regardless of propagation path, beam parameters, or receiver aperture size. The simulation parameters for each model are listed below.

\subsubsection{Small Aperture Model}

- $\lambda=632.8 \mathrm{~nm}$

- $z=100 \mathrm{~m}$

- $N=M=512$ pixels

- $w_{0}=.01 \mathrm{~m}$

- $\sigma_{f}=10^{-3} \mathrm{~m}$

- $\Delta x=\Delta y=0.19531 \mathrm{~mm}$

\subsubsection{Large Aperture Model}

- $\lambda=632.8 \mathrm{~nm}$

- $z=17,778 \mathrm{~m}$

- $N=M=800$ pixels

- $w_{0}=.2 \mathrm{~m}$ 
- $\sigma_{f}=1 \mathrm{~m}$

- $\Delta x=\Delta y=3.7 \mathrm{~mm}$

\subsubsection{Analysis}

The analysis begins by observing the average irradiance fluctuations of the GSM and FCS over different receiver apertures and turbulence strengths for the small aperture model in Figures 3.2-3.4. As expected in all cases the larger $\sigma_{g}^{2}$ is the closer the average irradiance of the GSM is to the average irradiance of a FCS.

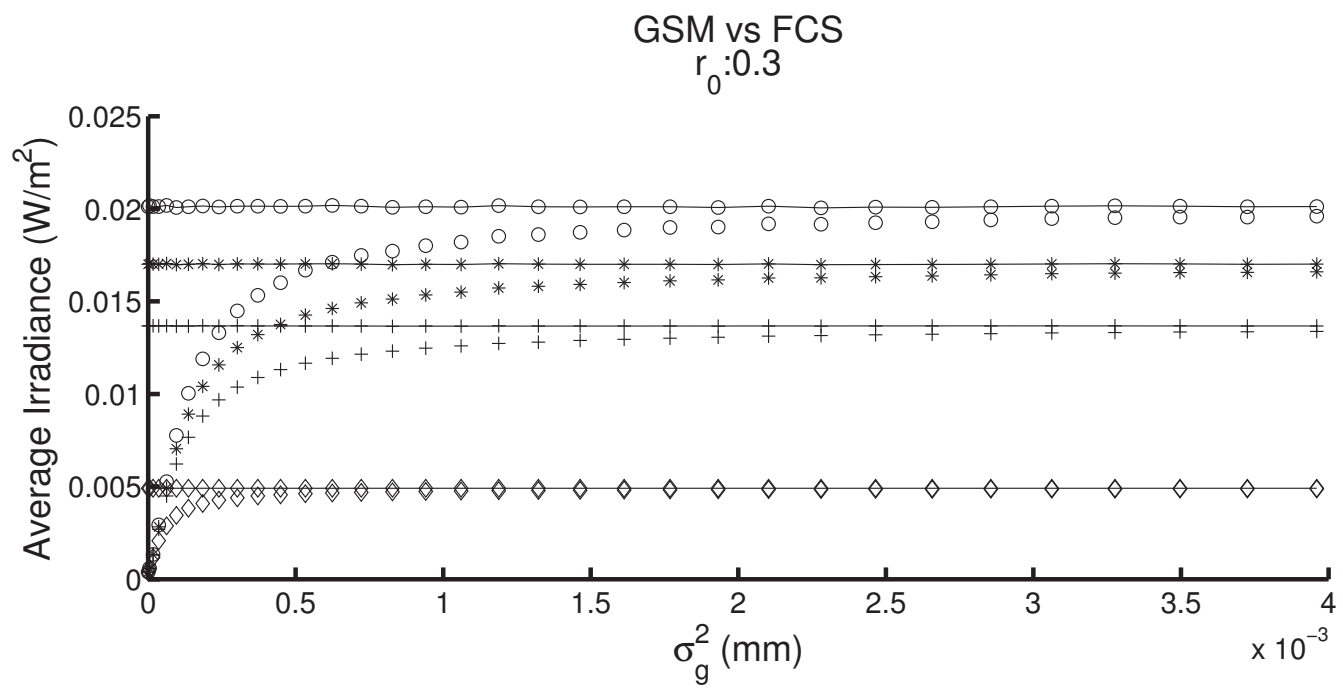

\begin{tabular}{|c|}
\hline GSM: Receiver Area $=4.8 \mathrm{e}-005 \mathrm{~m}^{2}$ \\
- FCS: Receiver Area $=4.8 \mathrm{e}-005 \mathrm{~m}^{2}$ \\
${ }^{*}$ GSM: Receiver Area $=1.1 \mathrm{e}-004 \mathrm{~m}^{2}$ \\
- FCS: Receiver Area $=1.1 \mathrm{e}-004 \mathrm{~m}^{2}$ \\
+ GSM: Receiver Area $=1.9 \mathrm{e}-004 \mathrm{~m}^{2}$ \\
+ FCS: Receiver Area $=1.9 \mathrm{e}-004 \mathrm{~m}^{2}$ \\
$\diamond$ GSM: Receiver Area $=7.7 \mathrm{e}-004 \mathrm{~m}^{2}$ \\
$\rightarrow$ FCS: Receiver Area $=7.7 \mathrm{e}-004 \mathrm{~m}^{2}$
\end{tabular}

Figure 3.2: Average received irradiance of increasing the spatial coherence of the GSM compared to a FCS through turbulence. [Small Aperture Model with $r_{0}=0.3$ ] 


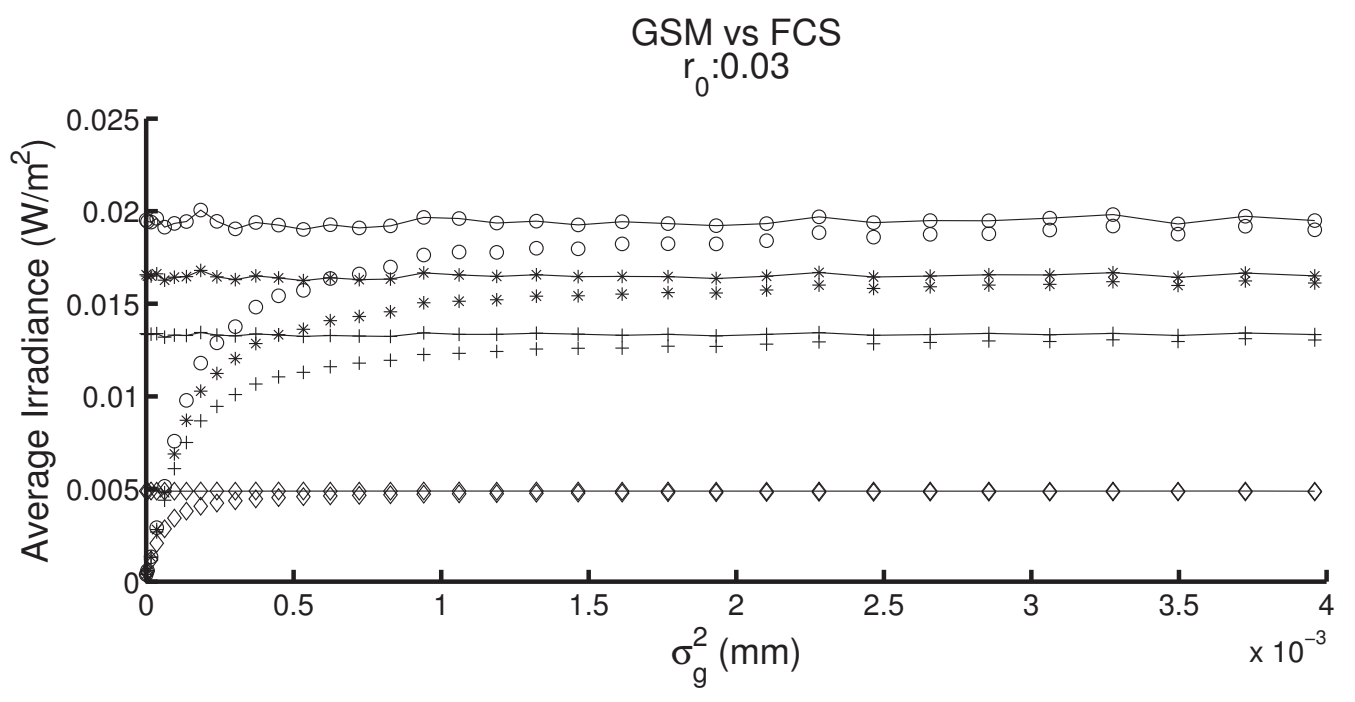

\begin{aligned}${ }^{\circ}$ GSM: Receiver Area $=4.8 \mathrm{e}-005 \mathrm{~m}^{2} \\ *$ FCS: Receiver Area $=4.8 \mathrm{e}-005 \mathrm{~m}^{2} \\ -$ GSM: Receiver Area $=1.1 \mathrm{e}-004 \mathrm{~m}^{2} \\ +$ FCS: Receiver Area $=1.1 \mathrm{e}-004 \mathrm{~m}^{2} \\ +$ GSM: Receiver Area $=1.9 \mathrm{e}-004 \mathrm{~m}^{2} \\ -$ FCS: Receiver Area $=1.9 \mathrm{e}-004 \mathrm{~m}^{2} \\ \rightarrow$ GSM: Receiver Area $=7.7 \mathrm{e}-004 \mathrm{~m}^{2} \\$\hline\end{aligned}

Figure 3.3: Average received irradiance of increasing the spatial coherence of the GSM compared to a FCS through turbulence. [Small Aperture Model with $r_{0}=$ $0.03]$

These results were obtained by calculating the average irradiance for 250 different realizations of $P C B(x, y)$ and $f c s(x, y)$ then averaging the individual means together. The purpose of multiple turbulence strengths, controlled by $r_{0}$, was to show that the upper-coherence limit will change with turbulence strength, yielding unique solutions for a particular channel. As expected the received average irradiance fluctuated more in the strong turbulence $\left(r_{0}=0.003 \mathrm{~m}\right)$, than the weak turbulence $\left(r_{0}=0.3 \mathrm{~m}\right)$.

In Figures 3.5-3.7 the averaged spatial variance for different GSM parameters 


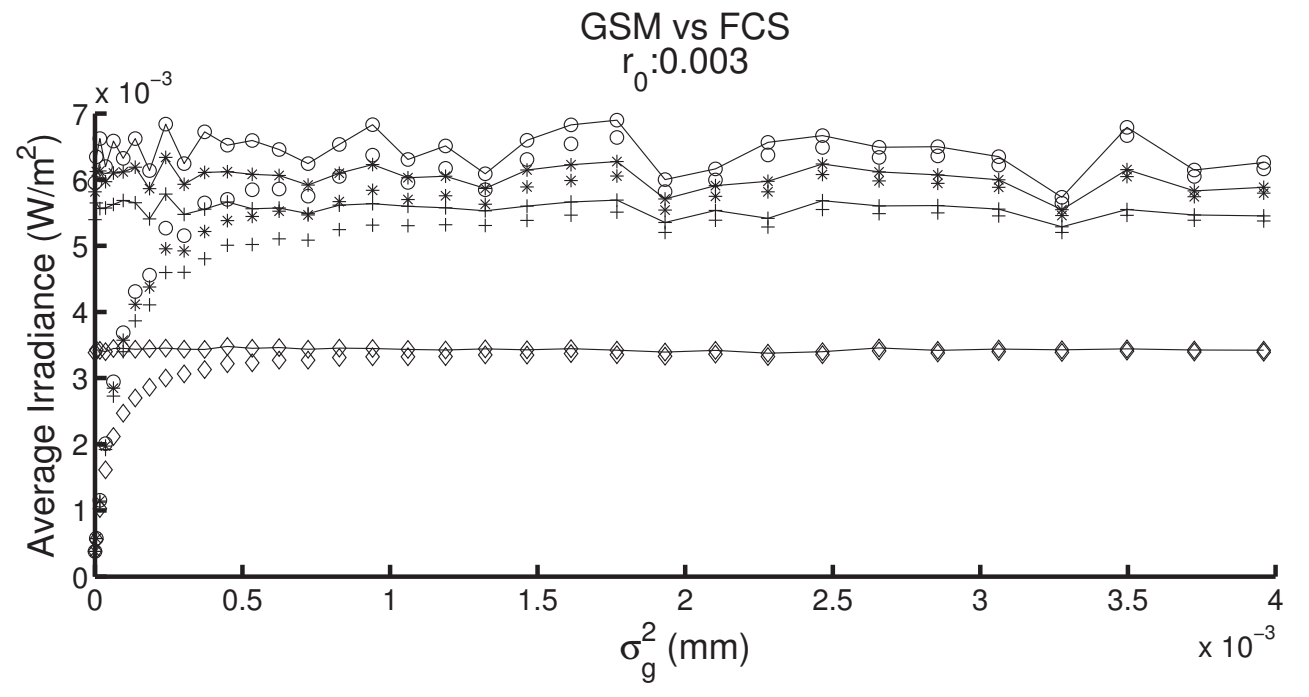

$\begin{array}{r}{ }^{-} \text {GSM: Receiver Area }=4.8 \mathrm{e}-005 \mathrm{~m}^{2} \\ { }^{*} \text { GCS: Receiver Area }=4.8 \mathrm{e}-005 \mathrm{~m}^{2} \\ \text { * } \text { FCS: Receiver Area }=1.1 \mathrm{e}-004 \mathrm{~m}^{2} \\ + \text { GSM: Receiver Area }=1.1 \mathrm{e}-004 \mathrm{~m}^{2} \\ + \text { FCS: Receiver Area }=1.9 \mathrm{e}-004 \mathrm{~m}^{2} \\ - \text { GSM: Receiver Area }=7.7 \mathrm{e}-004 \mathrm{~m}^{2} \\ \rightarrow \text { FCS: Receiver Area }=7.7 \mathrm{e}-004 \mathrm{~m}^{2} \\ \hline\end{array}$

Figure 3.4: Average received irradiance of increasing the spatial coherence of the GSM compared to a FCS through turbulence. [Small Aperture Model with $r_{0}=$ $0.003]$

are compared to the spatial variance of the FCS, where the horizontal dotted lines represent the maximum spatial variance of the FCS for each aperture value, the solid lines represent the average spatial variance of the FCS for each aperture value, and the dashed lines represent the minimum spatial variance of the FCS for each aperture value. It should be noted that the aperture area markers for the GSM correlate to the same aperture area for the horizontal line FCS data.

Inspection of Figures 3.5-3.7 show that as turbulence strength increases, the spatial variance of the FCS increases as shown by the widening of the minimum and 


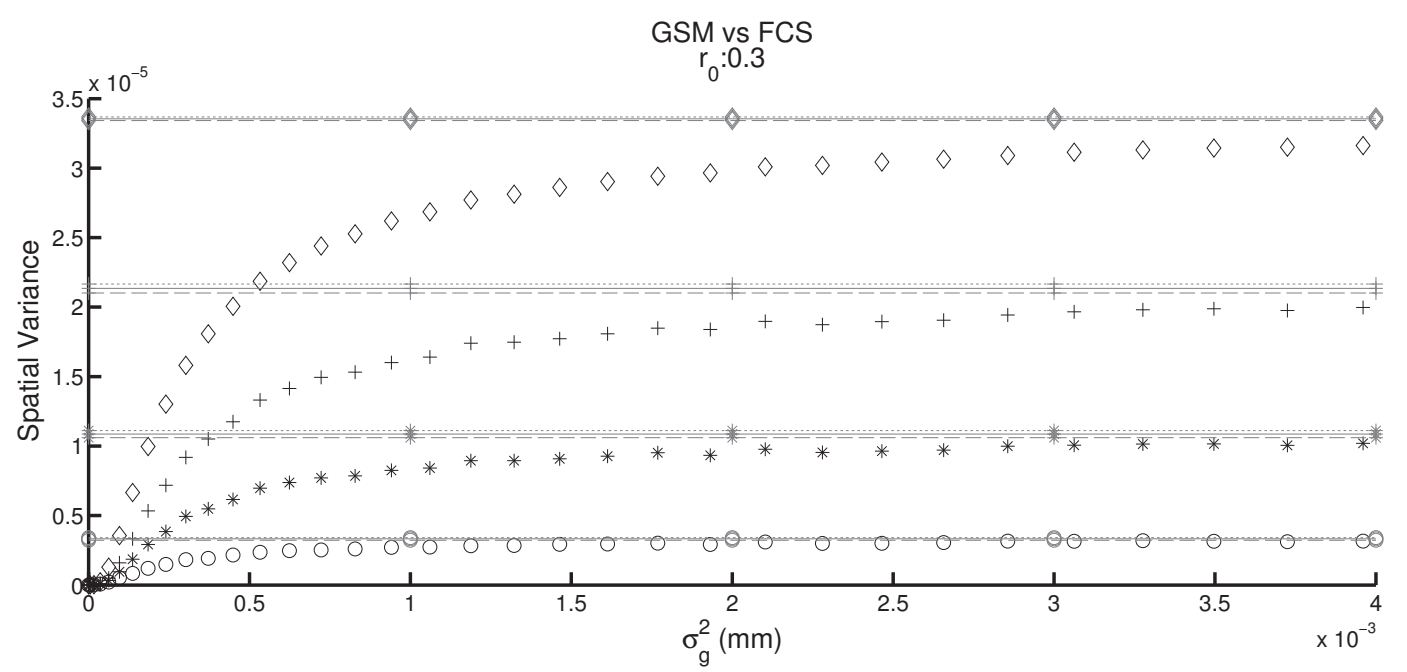

(a)

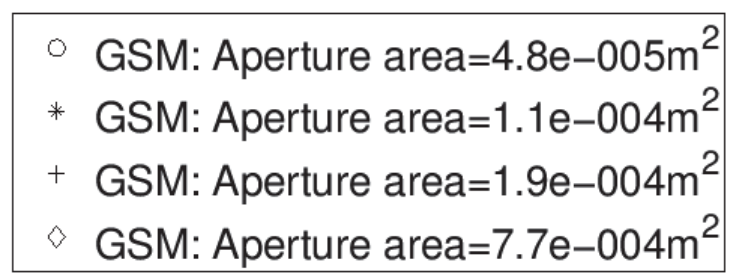

(b)

Figure 3.5: Spatial variance of GSM vs Spatial variance Statistics of Fully Coherent Source. [Small Aperture Model] The dotted line represents the maximum Spatial variance from the simulated FCS, the solid line represents the average spatial variance of the FCS, and the dashed line represents the minimum spatial variance of the FCS. The aperture size markers for the GSM and the FCS are the same to better allow comparison between models.

maximum lines. This spreading indicates that as turbulence strength increases the variance of the spatial variance increases with turbulence. In the weak turbulent regime(Figure 3.5) there are few if any variance encroachments, but in the stronger regimes(Figures 3.6 and 3.7), there are instances when the variance of the GSM becomes greater than the FCS. This result is expected since the larger $\sigma_{g}^{2}$ is, the the more coherent the propagating beam will become. It is for this reason that the UCL is being proposed for FSO communication that utilize PCBs. 


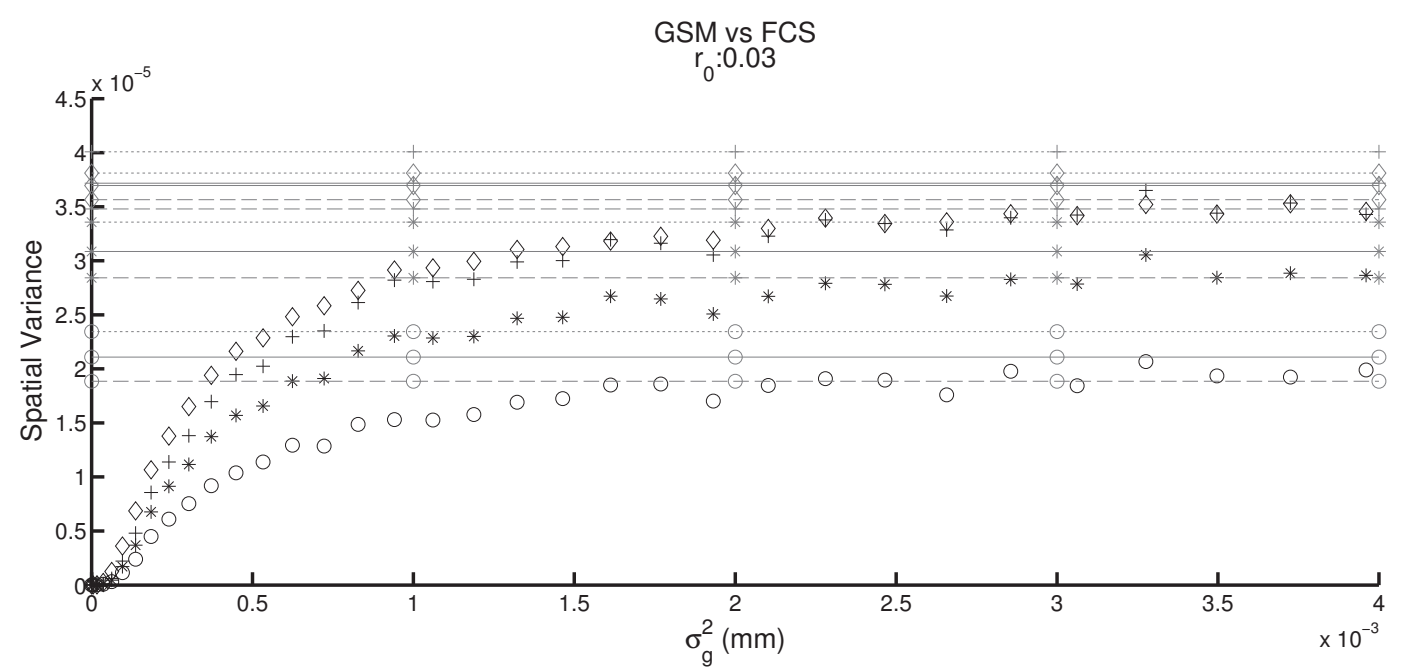

(a)

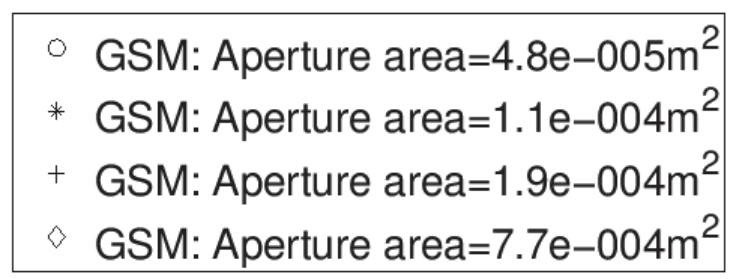

(b)

Figure 3.6: Spatial variance of GSM vs Spatial variance Statistics of Fully Coherent Source. [Small Aperture Model] The dotted line represents the maximum spatial variance from the simulated FCS, the solid line represents the average spatial variance of the FCS, and the dashed line represents the minimum spatial variance of the FCS. The aperture size markers for the GSM and the FCS are the same to better allow comparison between models.

The next step is to analyze the scintillation index $(S I)$ to determine the optimal UCL. For reference the definition of $S I$ is

$$
S I=\frac{\operatorname{variance}(q)}{[\operatorname{mean}(q)]^{2}}
$$

where $q$ in this instance is a 2D matrix of the apertured simulated irradiance for one instance of $P C B(x, y)$ or $f c s(x, y)$. The UCL is defined as the spatial coherence cut-off point, which is found by determining the $-3 \mathrm{~dB}$ point from the maximum 


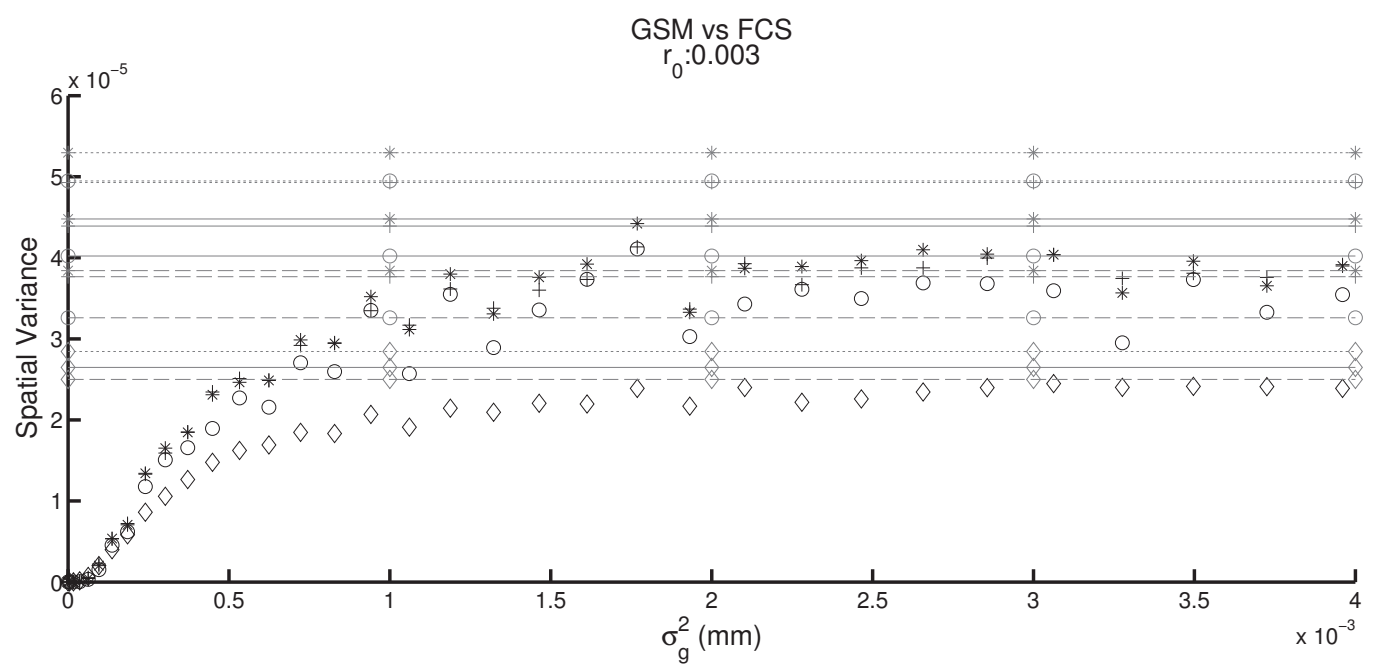

(a)

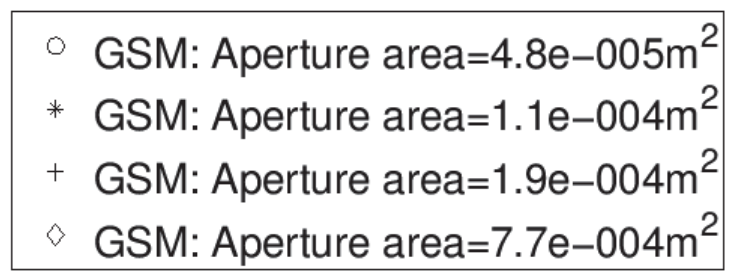

(b)

Figure 3.7: Spatial variance of GSM vs Spatial variance Statistics of Fully Coherent Source. [Small Aperture Model] The dotted line represents the maximum spatial variance from the simulated FCS, the solid line represents the average spatial variance of the FCS, and the dashed line represents the minimum spatial variance of the FCS. The aperture size markers for the GSM and the FCS are the same to better allow comparison between models.

value of $S I_{d B}$. This $-3 \mathrm{~dB}$ point corresponds to the approximate half power of the transmitting GSM beam when compared to a FCS. The $S I$ equation in decibels $(\mathrm{dB})$, is defined as

$$
S I_{d B}=10 \log _{10}\left(\frac{S I(P C B)}{\langle S I(f c s)\rangle}\right)
$$

where $\langle S I(f c s)\rangle$ denotes the average of $S I(f c s)$. The averaging was done to consolidate all of the FCS data from Figures 3.5-3.7 to better estimate the half power 
point of a PCB when compared to a FCS. These results are now presented in Figures 3.8-3.10, with the UCL being represented by the intersection of $S I_{d B}$ and the dotted line.

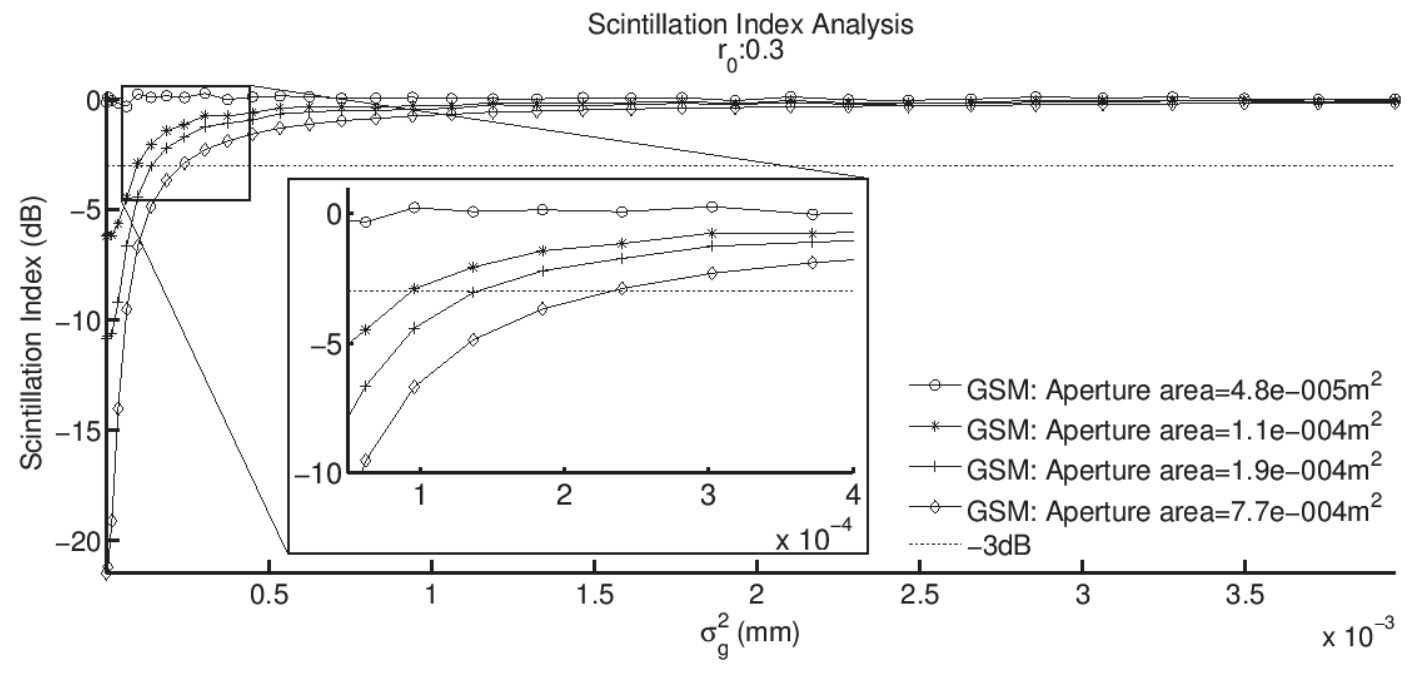

Figure 3.8: Scintillation Index of GSM. [Small Aperture Model]

As observed in Figures 3.8-3.10, when turbulence strength increases $S I_{d B}$ starts to converge to the same UCL value, regardless of aperture size. In weak turbulence the smallest aperture size, basically a single point, has the best response. The reason for this is because the point is on-axis, there is minimal turbulence to refract it, and the more incoherent the beam is, the more uniform the resulting intensity will be. The more interesting results appear in Figure 3.9 and Figure 3.10, where it is shown that as the turbulence strength increases the UCL begins to converge to the same value, regardless of aperture size.

Figures 3.11-3.12 show the $S I_{d B}$ data for larger aperture sizes as defined in the large aperture model. As expected, the large aperture results take on the same form as the small aperture results. The large aperture $S I_{d B}$ data has been shifted by the maximum value of $S I_{d B}$ to zero dB to make the UCL value more obvious. These large aperture results coincide with the small aperture model, showing that a UCL 


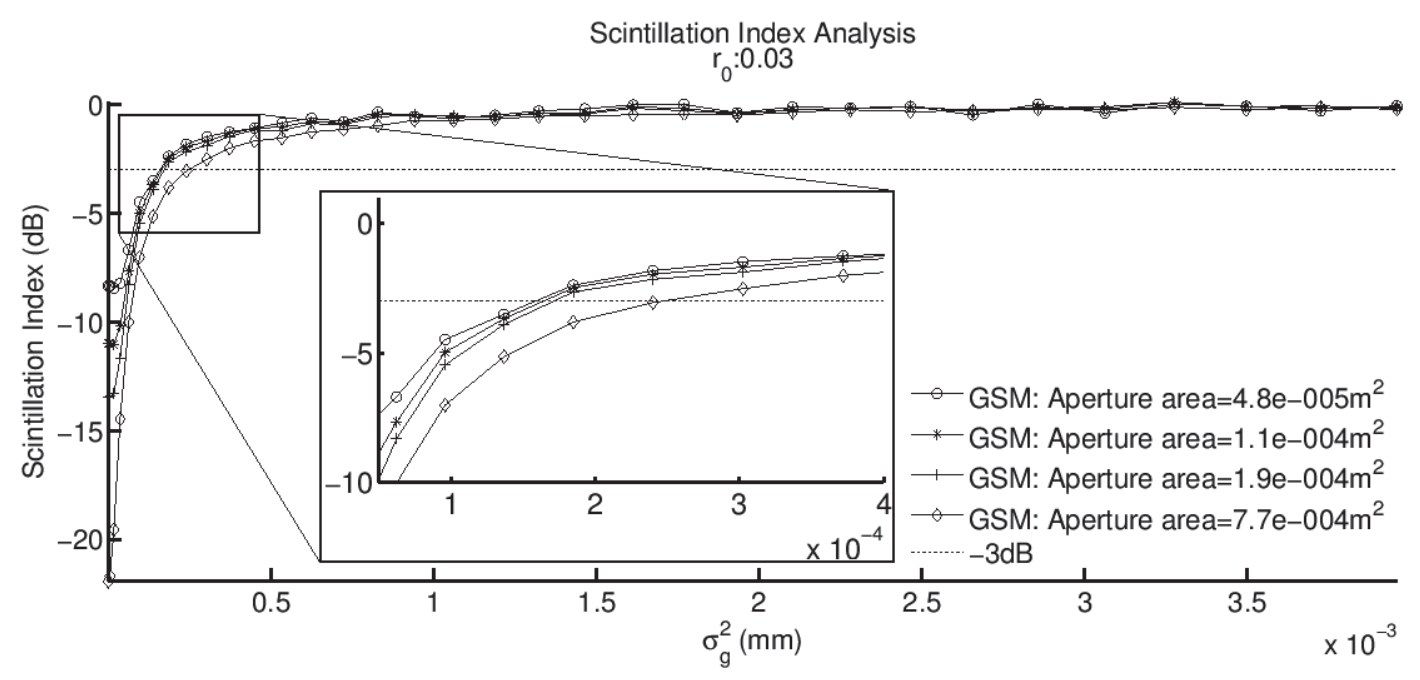

Figure 3.9: Scintillation Index of GSM. [Small Aperture Model]

is theoretically obtainable regardless of aperture size or turbulence strength.

\subsection{Conclusions}

This chapter has shown that there is a straight forward method to calculate the upper-spatial correlation width of a GSM beam in a closed channel, regardless of aperture size, or turbulence strength. This has been shown through the unique solutions to the UCL for each simulated channel. By using this analysis method to find a limit, it takes some of the guess work out of determining the correct PCB parameters, which will help simplify correcting for turbulence. Future work that needs to be completed is verifying the UCL experimentally, and ensuring that the UCL is obtainable in the Fraunhoffer domain.

An off the shelf experiment to test the UCL theory could be done using a spatial light modulator to create the GSM, with the control computer linked to the receiver through an Ethernet connection. The receiver could be as simple as an Ethernet camera or more complex with a camera and computer setup. 


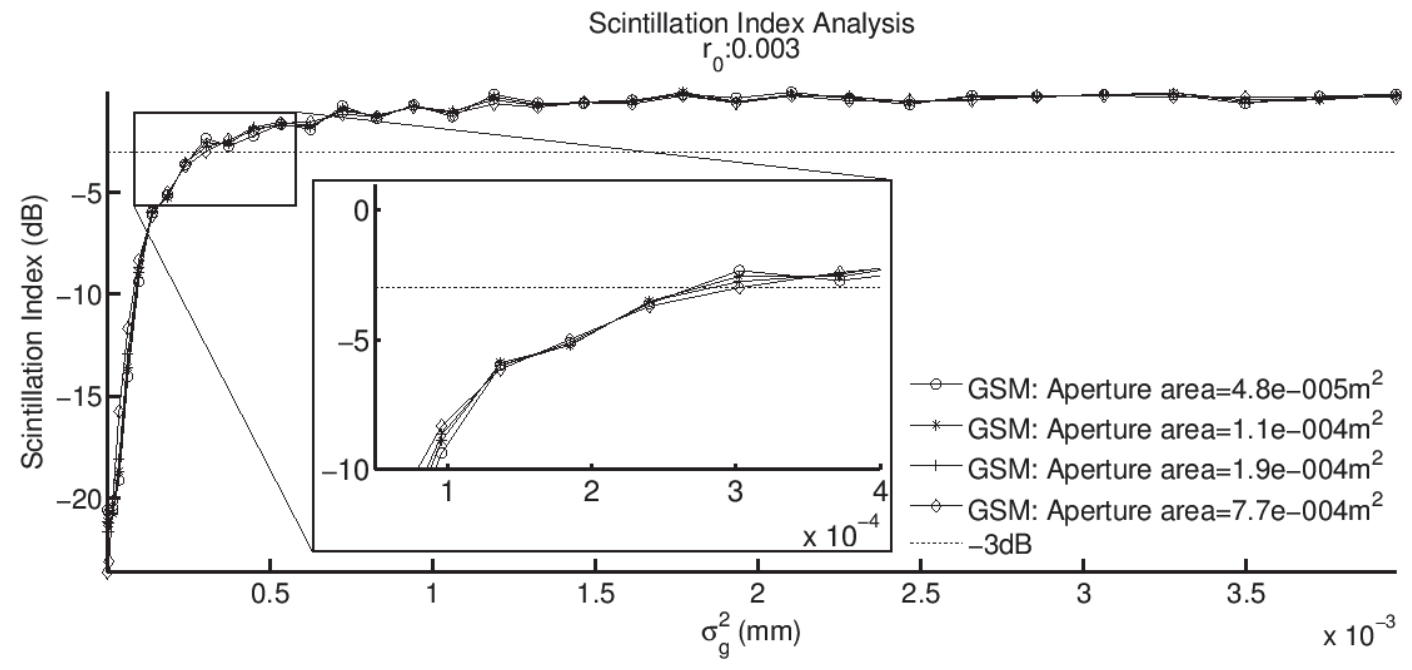

Figure 3.10: Scintillation Index of GSM. [Small Aperture Model]

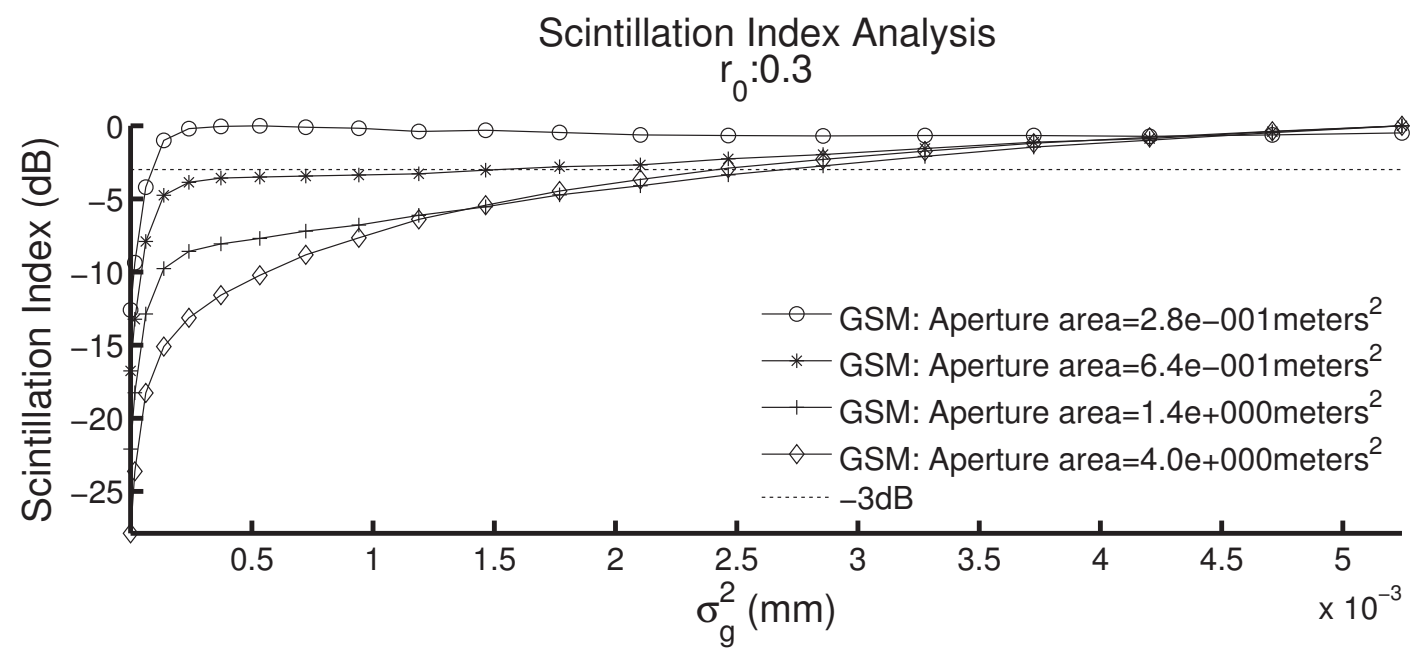

Figure 3.11: Scintillation Index of GSM. [Large Aperture Model] 


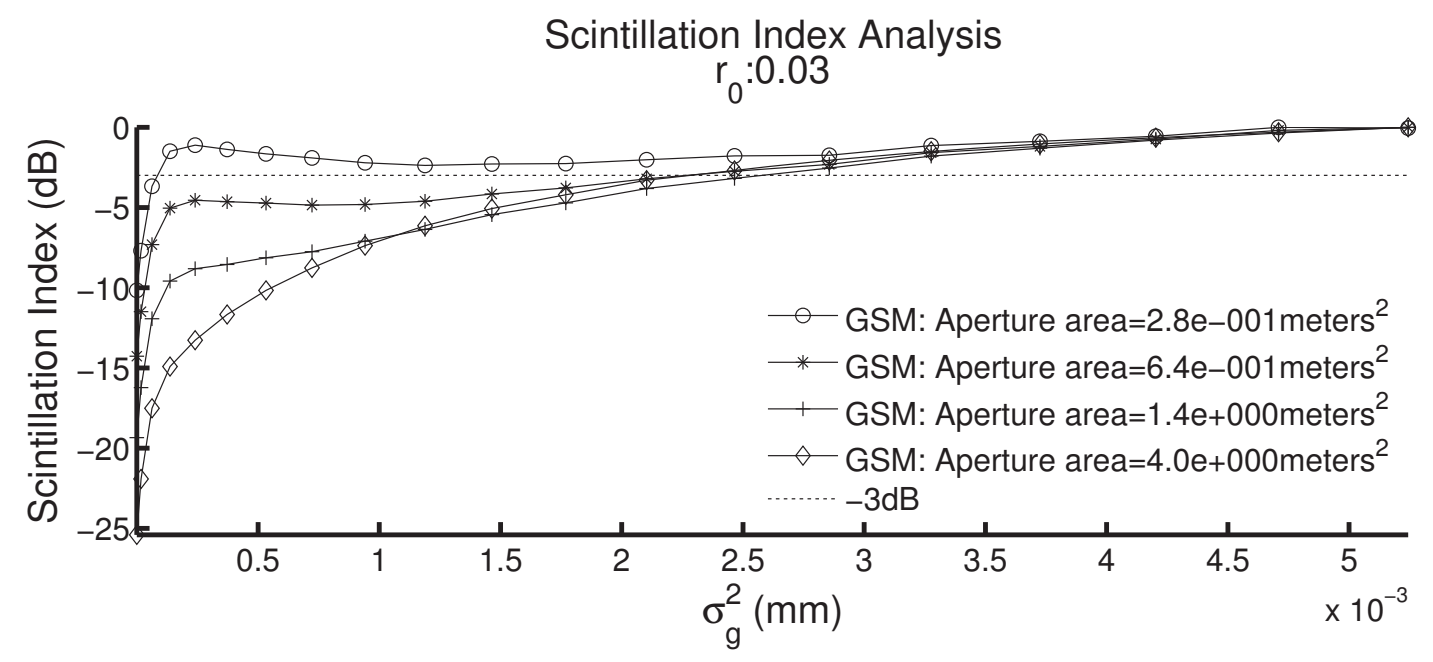

Figure 3.12: Scintillation Index of GSM. [Large Aperture Model] 


\section{Chapter 4}

\section{A Technique for Estimating the \\ Number of Control Elements}

needed to Approximate the

\section{Gaussian-Schell model in a Free}

\section{Space Optical Communications}

\section{Channel}

\subsection{Introduction}

Traditional free space optical(FSO) communications are adversely affected by turbulence, causing speckling in the received field under many operating conditions of potential interest. ${ }^{1-6}$ Recent work has shown that transmitting a partially coherent beam has promise to mitigate some of the speckling that occurs at the receiver 
without significantly reducing the signal-to-noise ratio(SNR) of the received signal ${ }^{6,8-11,13,13-17}$. Most of the work done in this area has focused on the concepts of the propagating field, and less on analyzing what is necessary to bring the concept to practice. This chapter presents the results of a wave optics simulation designed to determine the minimum number of elements necessary in a spatial light modulator to generate a useful approximation of an ideal Gaussian-Schell model(GSM) beam $^{18,19}$. The goal was to determine the minimum number of elements necessary to stabilize the scintillation index(SI) of the GSM at the receiver.

This chapter shows that the spatial resolution available in the current generation of commercial SLMs is sufficient to generate a GSM beam through turbulence. The fewer elements in a SLM the more likely the received field will be farther from the ideal GSM. This chapter estimates the point in which increasing the number of elements in a SLM leads to diminishing returns in stabilizing the received field.

The rest of the chapter is organized as follows. Section 4.2 is an overview of the split-step method for modeling the GSM beam through a horizontal turbulence channel. In section 4.3 the simulation parameters and results will be presented and discussed. Finally, section 4.4 will end with the conclusion and thoughts about future work.

\subsection{Mathematical Model}

This chapter uses the split-step method of modeling ${ }^{35,36}$ to simulate propagation of the GSM beam though turbulence to the receiver. The first step is to generate a Gaussian beam, this beam is then combined with the phase control screen that would be applied to the SLM. The resulting function, $E_{s l m}$, is the simulated light

off of the SLM, this reflected light is defined as one instance of a GSM beam before 
propagation. The remaining instances of a fully developed GSM beam are filled in by other independently generated phase screens at the rate that the SLM can apply them.

The simulation then propagates one GSM instance through a number of turbulence phase screens to a final receiver distance, z. All propagation path lengths, $\Delta z$, are equal to simplify the scaling of the propagation mathematics ${ }^{35}$. The main problem with this turbulence correction technique is the reliance on the frame rate of the implementing SLM, this is because the frame rate of the SLM should be greater than the rate of change of the atmosphere. An overview of this method for generating one instance of the GSM is presented in Figure 4.1.

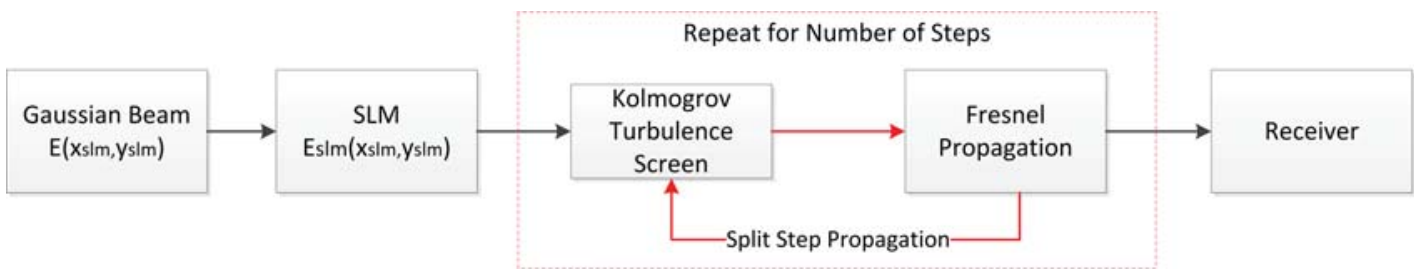

Figure 4.1: Overview of split-step simulation method for one instance of the GSM.

One instance of the GSM of a partially coherent beam reflected from the spatial light modulator before atmospheric propagation is given by

$$
E_{s l m}\left(x_{s l m}, y_{s l m}\right)=E\left(x_{s l m}, y_{s l m}\right) \exp \left[j \theta\left(x_{s l m}, y_{s l m}\right)\right]
$$

where $x_{s l m}$ and $y_{s l m}$ are the number of movable elements in the $x$ and $y$ directions of the spatial light modulator respectively, $E\left(x_{s l m}, y_{s l m}\right)$ is the Gaussian beam incident upon the SLM defined by ${ }^{29}$

$$
E\left(x_{S L M}, y_{S L M}\right)=\exp \left[-\frac{\left(x_{S L M} \Delta x_{a t m o}\right)^{2}+\left(y_{S L M} \Delta y_{a t m o}\right)^{2}}{w_{0}^{2}}\right]
$$


where $\Delta x_{a t m o}$ and $\Delta y_{a t m o}$ are the sample spacings of the atmosphere in the $x$ and $y$ directions respectively, $w_{0}$ is the $e^{-1}$ radius of the beam, and $\theta\left(x_{S L M}, y_{S L M}\right)$ is the phase control screen of the GSM applied to the SLM, which is defined as

$$
\theta\left(x_{S L M}, y_{S L M}\right)=\left[f\left(x_{S L M}, y_{S L M}\right) \otimes r\left(x_{S L M}, y_{S L M}\right)\right]\left(\Delta x_{a t m o} \Delta y_{a t m o}\right)
$$

where $\otimes$ represents the two dimensional convolution operator, with $f\left(x_{S L M}, y_{S L M}\right)$ and $r\left(x_{S L M}, y_{S L M}\right)$ being defined as

$$
\begin{gathered}
f\left(x_{S L M}, y_{S L M}\right)=\frac{1}{2 \pi \sigma_{f}^{2}} \exp \left[-\frac{\left(x_{S L M} \Delta x_{a t m o}\right)^{2}+\left(y_{S L M} \Delta y_{a t m o}\right)^{2}}{2 \sigma_{f}^{2}}\right] \\
r\left(x_{S L M}, y_{S L M}\right)=\frac{\left(\gamma\left(x_{S L M}, y_{S L M}\right)-.5\right) \sigma_{r}}{\left(\Delta x_{a t m o} \Delta y_{a t m o}\right)^{2}} .
\end{gathered}
$$

$\gamma\left(x_{S L M}, y_{S L M}\right)$ is a matrix of numbers provided by a random number generator whose output is uniformly distributed on $[0,1], \mathscr{F}$ represents the Fourier transform, $\sigma_{r}$ relates to the amplitude variation of the phase screen, and $\sigma_{f}$ is the transverse spatial correlation length. Both $\sigma_{r}$ and $\sigma_{f}$ have units of length.

The spatial correlation width, $\sigma_{g}^{2}$, of $\theta$ is ${ }^{9,13}$

$$
\sigma_{g}^{2}=\frac{8 \pi \sigma_{f}^{4}}{\sigma_{r}^{2}}
$$

Physically $\sigma_{g}^{2}$ is a measure of the $\mathrm{e}^{-1}$ radius of the correlation function; more importantly it is a measure of the spatial coherence of the beam.

The first step to propagate $E_{S L M}$ through atmospheric turbulence is to downsample $\theta$ from the maximum spatial resolution, to the desired number of pixels in 
the simulated SLM. This is done by linearly sampling $\theta\left(\max \left(x_{S L M}\right), \max \left(y_{S L M}\right)\right)$ every $\frac{\max \left(x_{S L M}\right)}{x_{S L M}}$ and $\frac{\max \left(y_{S L M}\right)}{y_{S L M}}$. Now the downsampled $\theta$ values need to be replicated to achieve a uniform oversampling of $\theta$ to match the atmospheric sampling for simulating the SLM. This ensures that the atmospheric sampling is consistent for every simulated case by oversampling the field when the SLM has few phase control elements. An example with $x_{S L M}=y_{S L M}=[10,800]$ has been given in Figure 4.2.

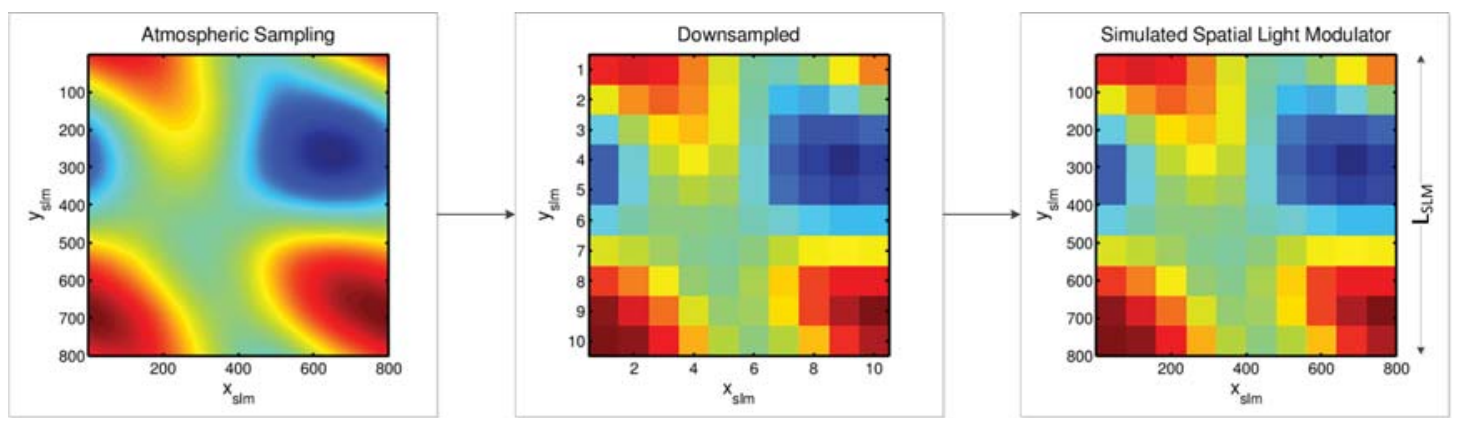

Figure 4.2: First stage is to sample $\theta$ at atmospheric resolution. The second stage is to downsample $\theta$ to the simulated spatial resolution of the SLM. The third stage is to replicate the values to achieve an oversampling that matches the atmospheric sampling.

$E_{S L M}$ is then symmetrically zero padded to the same sampled size as the turbulence channel, $x_{a t m o}$ and $y_{a t m o}$. A physical image of how $E_{S L M}$ resides centered in the turbulence channel is presented in Figure 4.3.

After padding $E_{S L M}$, a Kolmogorov turbulence phase screen, $T\left(x_{a t m o}, y_{a t m o}\right)$, is generated by MatLAB's adaptive optics toolbox and placed in the atmospheric channel of the GSM field before propagating. Mathematically this operation is defined as $E_{S L M}\left(x_{a t m o}, y_{a t m o}\right) \mathrm{e}^{j T\left(x_{a t m o}, y_{a t m o}\right)}$. The turbulence strength for this simulation is controlled through the Fried parameter, $r_{0}$, defined by ${ }^{7}$

$$
r_{0}=0.185\left[\frac{4 \pi^{2}}{k^{2} C_{n}^{2} \Delta z}\right]^{3 / 5},
$$



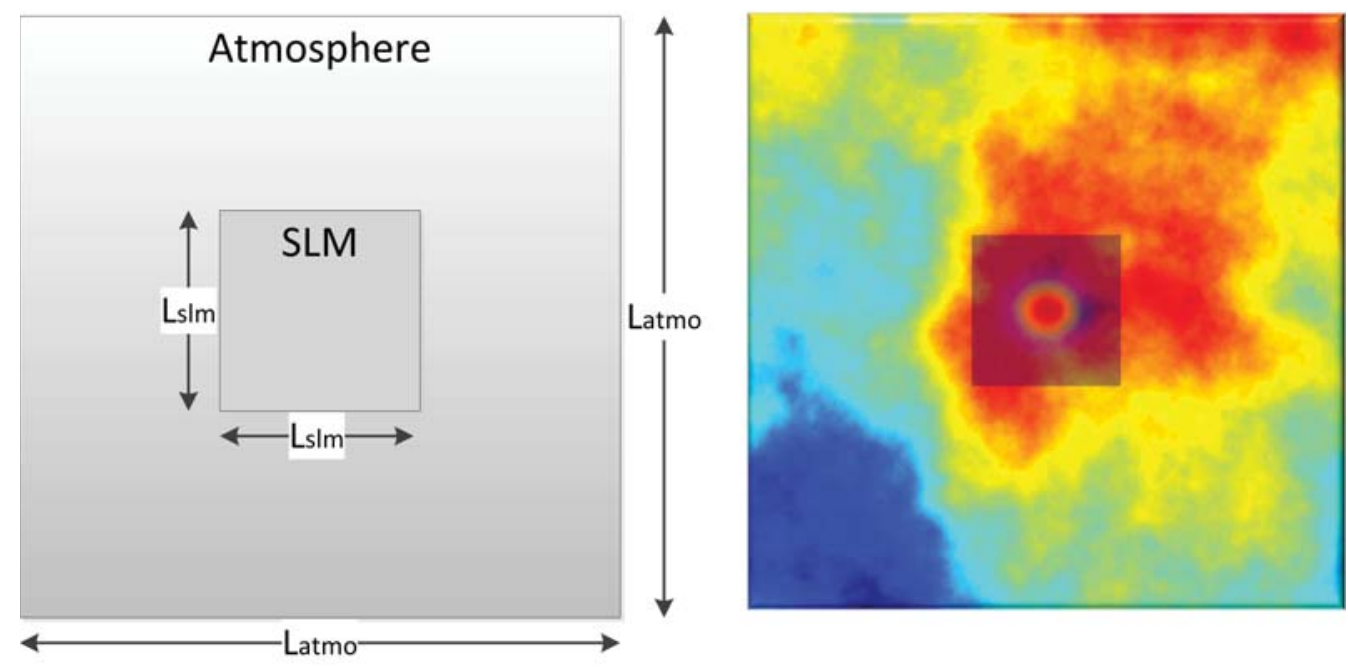

Figure 4.3: (Left) Physical comparison of SLM and the atmospheric channel. (Right) Simulated SLM and atmospheric channel overlay at $z=0$.

where $C_{n}^{2}$ is the atmospheric structure constant, $k$ is the scalar wave number defined as $k=2 \pi / \lambda$, and $\Delta z$ is the propagation distance between turbulence layers, defined as

$$
\Delta z=\frac{z}{A_{s}}
$$

where $z$ is the total propagation distance, and $A_{s}$ is the number of atmospheric slices to compute.

The field is then propagated using the Fresnel propagator ${ }^{6}$ as defined by

$$
H(u, v)=\mathscr{F}\left\{\frac{\exp [j k \Delta z]}{j \lambda \Delta z} \exp \left[\frac{j k}{2 \Delta z}\left(\left(x_{\text {atmo }} \Delta x_{\text {atmo }}\right)^{2}+\left(y_{\text {atmo }} \Delta y_{\text {atmo }}\right)^{2}\right)\right]\right\}
$$

where $u$ and $v$ are the spatial frequencies of the propagator, and $\lambda$ is the wavelength. 
Mathematically the first propagation is defined as

$$
\mathbb{F}\left\{E_{\text {slm }}\left(x_{\text {atmo }}, y_{\text {atmo }}\right) \mathrm{e}^{j T\left(x_{a t m o}, y_{a t m o}\right)}\right\} H(u, v) .
$$

After propagation another independent turbulence screen with the same statistics is placed in the atmospheric channel and propagation occurs again. This process is repeated $A_{s}$ times. This entire process is then repeated, $S$ times, to achieve the desired number of instances that compose the fully developed GSM, as shown in Figure 4.4.

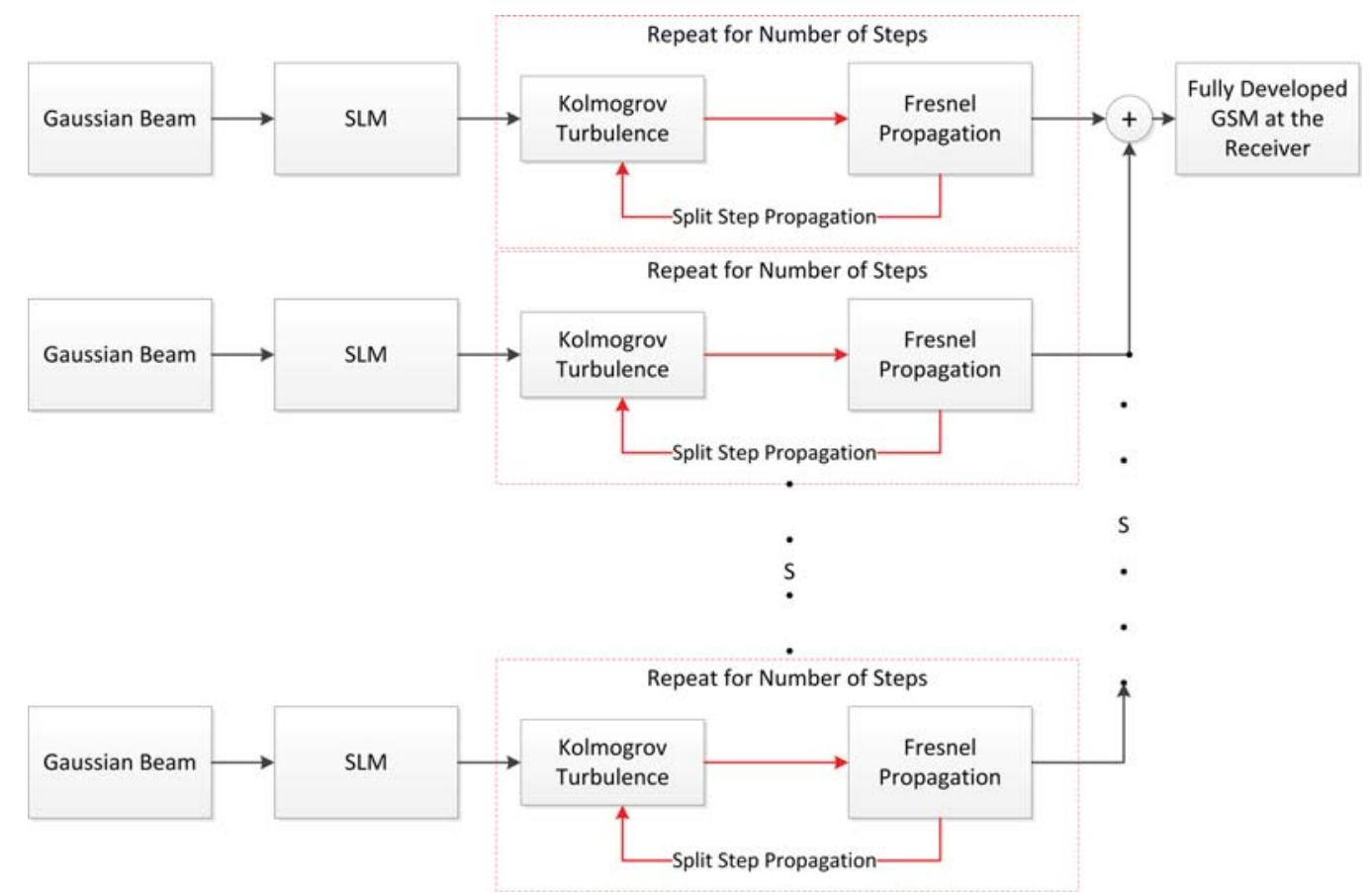

Figure 4.4: Overview of split-step simulation method to fully develop the GSM. 


\subsection{Simulation Results}

The simulation results are for finite apertures of diameter D, with the same physical simulation propagation paths, while the number of phase control elements in the spatial light modulator change. To compensate for the static physical dimensions, the elements themselves are assumed to be physically scaled as shown in Figure 4.2, to ensure that the results correspond to number of elements, keeping the total physical length of the SLM, $L_{S L M}$, and atmosphere, $L_{a t m o}$, constant. The simulation parameters are listed below.

- $\lambda=632.8 \mathrm{~nm}$

- $w_{0}=0.05 \mathrm{~m}$

- $\Delta z=1,000 \mathrm{~m}$

- $z=4,000 \mathrm{~m}$

- $x_{S L M}=y_{S L M}=[4,5,8,10,20,40,50,100,200,400,800]$ pixels

- $L_{S L M}=.2 \mathrm{~m}$

- $x_{\text {atmo }}=y_{\text {atmo }}=4,000$ pixels

- $L_{\text {atmo }}=1 \mathrm{~m}$

- $\sigma_{f}=0.04 \mathrm{~m}$

- $\sigma_{g}=0.005 \mathrm{~m}$

- $S=5,000$

It should be noted that the results of this chapter use a high number of GSM instances, $S$, when calculating the SI. This was done to work in the limit and ensure 
that the GSM was fully developed at the receiver. In a practical FSO communications system $S$ would be significantly reduced.

The analysis begins with a low turbulence model as shown in Figure 4.5. The goal is to determine stability and linearity of the SI at the receiver. Analyzing the scintillation index shows that regardless of the receiver diameter, D, in a low element SLM there is a significant non-linear component in the received SI, and as the number of elements increases the scintillation index values stabilize. This trend continues in both Figures 4.6 and 4.7 regardless of aperture size or turbulence strength. This is expected with this technique since the fewer elements in a SLM, the less developed the propagating GSM beam will be.

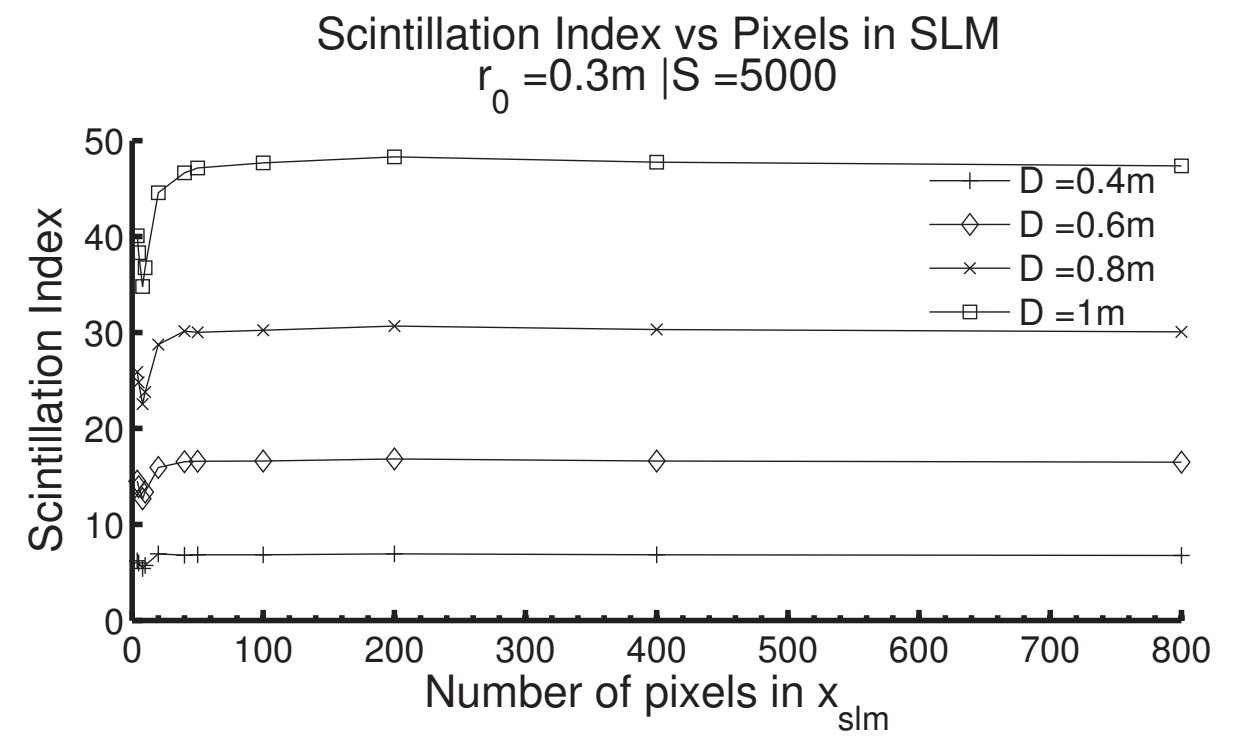

Figure 4.5: Scintillation Index vs Controllable Pixel Elements, Fried Parameter = $0.3 \mathrm{~m}$

The number of elements necessary to compensate for atmospheric turbulence is turbulence dependent as shown by observing the varying degree that the non-linear term lasts in the scintillation index before stabilizing. This is not necessarily an issue since in all cases by the 50 by 50 element SLM the scintillation index stabilized. The 


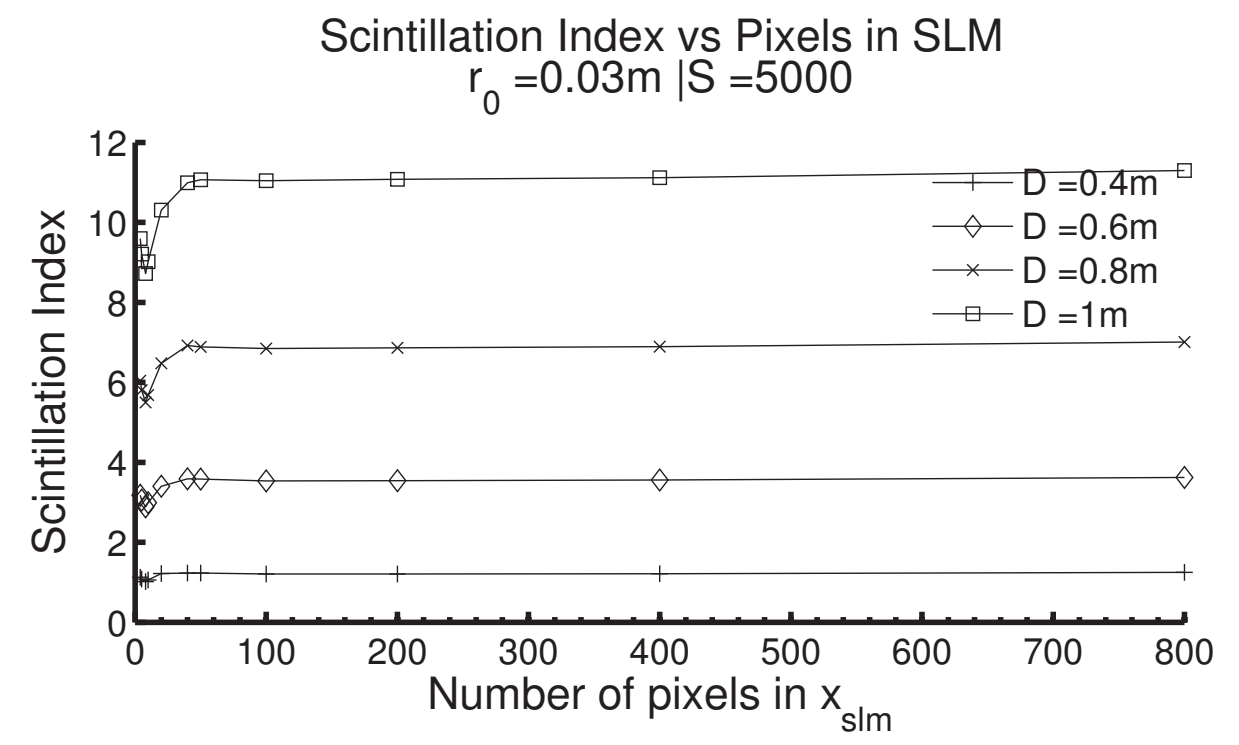

Figure 4.6: Scintillation Index vs Controllable Pixel Elements, Fried Parameter $=$ $0.03 \mathrm{~m}$

reason for this being a non-issue, is that SLM's with more elements than this are commonly available in off the shelf products by Boulder Nonlinear, Iris AO, and Holoeye Photonics AG. This means that the limiting factor to implement the GSM in FSO communications is the frame rate of the SLM.

\subsection{Conclusion}

It has been shown that the number of elements necessary to correct for atmospheric turbulence and generate a GSM at the receiver is turbulence dependent. Fortunately though the minimum number of elements necessary to correct for the strongest simulated turbulence case is only a 50 by 50 element spatial light modulator, since these types of devices are already commercially available the only real problem remaining is to increase the switching frequency that these SLMs operate at, allowing more frames to be generated in a smaller time frame, and allowing the GSM to 


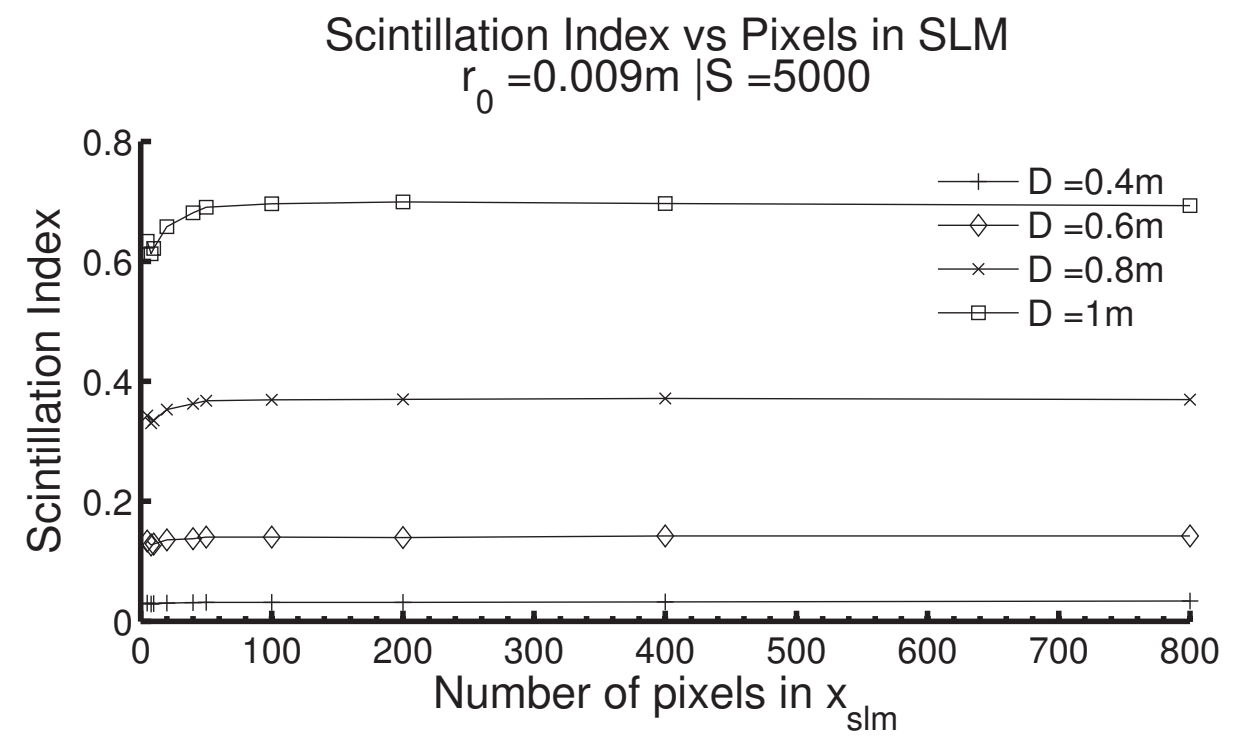

Figure 4.7: Scintillation Index vs Controllable Pixel Elements, Fried Parameter $=$ $0.009 \mathrm{~m}$

become a practical method for mitigating turbulence. 


\section{Chapter 5}

\section{Conclusion}

\subsection{Summary}

This dissertation has shown that the Gaussian-Schell model of partial coherence is a useful and straight forward method to mitigate atmospheric turbulence by controlling the statistics of the source to reduce the variance of the received field while maintaining the SNR. This means that traditional expensive adaptive optics are not necessary in a FSO system to compensate for atmospheric turbulence. This concept has been experimentally demonstrated in the far-field using a liquid crystal SLM over a range of turbulence strengths.

A method for determining optimal GSM beam parameters in an unknown turbulent atmosphere was developed and modeled using a wave optics simulation. The results from this work show that it is possible to create a partially coherent source and a fully coherent source using the same hardware to determine optimal transmission parameters in otherwise unknown turbulence while maintaining SNR. This technique has also been shown to work for a variety of turbulence strengths, atmospheric cross-sections, and receiver diameters. The only added complication is that 
it requires a simple RF link to close the channel. Fortunately though no critical information is transfered over the RF link helping to ensure secure optical communications.

Finally it has been shown that generating a fully controllable GSM using a SLM to correct for atmospheric turbulence is close to being a practical solution. This is due to the high spatial resolution of the current generation of spatial light modulators. The main problem with generating a GSM beam is the low frame rate of the implementing SLM, which will be solved as companies slowly increase the frame rate with new technology and techniques.

\section{$5.2 \quad$ Future Work}

Future work that needs to be completed in this area is to prove the UCL experimentally. This would be a fairly easy experiment to setup using a spatial light modulator and fully coherent source for transmission. The receiver could be a simple wireless webcam that would operate over WiFi to collect the data. Only one computer would be required which would control the SLM, and analyze the data, since the closed loop could be completed using a local area network solution instead of the dedicated RF link in this controlled experiment.

Another approach would be to develop a set of modified screens that that are optimized to reduce the number of GSM instances required to approach the ideal GSM intensity pattern. This work would be particularly useful due to the slow nature of the current generation of spatial light modulators. 


\section{Bibliography}

[1] Special Issue on laser speckle, Journal of the Optical Society of America, 1976; 66.

[2] Dainty J. The statistics of Speckle Patterns, Progress in Optics, 1976; 14:1-46.

[3] Clifford S. The Classical theory of Wave Propagation in a Turbulent Medium. In: Strohbehn, J editor. Laser beam propagation in the Atmosphere. Heidelberg: Springer-Verlag; 1978. p. 9-43.

[4] Goodman J. Statistical Properties of Laser Speckle Patterns. In: Dainty, J.C., editor. Laser Speckle and Related Phenomena. Berlin: Springer-Verlag; 1984. p. $9-75$.

[5] Chernov L. Wave Propagation in a Random Medium. New York(NY): McGrawHill; 1960. p. 59-124.

[6] Goodman J. Statistical optics. New York(NY): Wiley; 1985.

[7] Roggemann M, Welsh B, Imaging through turbulence. 1st ed. Boca Raton: The CRC Press; 1996.

[8] Ricklin J, Davidson F. Atmospheric turbulence effects on a partially coherent Gaussian beam: implications for free-space laser communication. Journal of the Optical Society of America. 2002; 19:1794-1802. 
[9] Xiao X, Voelz D. Wave optics simulation approach for partial spatially coherent beams. Optics Express. 2006; 14:6986-6992.

[10] Ricklin J, Davidson F. Free-space laser communication using a partially coherent laser source. Proceedings of SPIE. 2002; 4538:13-23.

[11] Voelz D, Xiao X. Metric for optimizing spatially partially coherent beams for propagation through turbulence. Optical Engineering. 2009; 48.

[12] Berman G, Bishop A, Chernobrod B, Gorshkov V, Lizon D. Reduction of laser intensity scintillations in turbulent atmospheres using time averaging of a partially coherent beam. Journal of Physics B. 2009; 42(5).

[13] Drexler K, Roggemann M, Voelz D. Use of a partially coherent transmitter beam to improve the statistics of received power in a free-space optical communication system: theory and experimental results. Optical Engineering. 2011; 50.

[14] Drexler K, Roggemann M. Far-field scintillation reduction utilizing GaussianSchell model beams. OSA Technical Digest. 2011; LTuD2.

[15] Drexler K, Roggemann M. Method for determining the spatial correlation width of a Gaussian-Schell beam to optimize the scintillation index over long horizontal turbulent paths. Optical Engineering. 2012; 51.

[16] Korotkova O, Andrews L, Phillips R. Model for a partially coherent Gaussian beam in atmospheric turbulence with application in Lasercom. Optical Engineering. 2004; 43:330-341.

[17] Korotkova O, Andrews L, Phillips R. Speckle Propagation through Atmospheric 
Turbulence: Effects of a Random Phase Screen at the Source. SPIE Proceedings. 2002; 4821:98-109.

[18] Mandel L, Wolf E. Optical coherence and quantum optics. 1st ed. Cambridge(NY): Cambridge University Press. 1995.

[19] Andrews L, Phillips R. Laser beam Propagation through Random Media. 1st ed. Bellingham(WA): SPIE Press; 2005. p. 667-704

[20] Piquero G, Gori F, Romanini P, Santarsiero M, Borghi R, Mondello A. Synthesis of partially polarized gaussian schell-model sources. Optics Communications. $2002 ; 208: 9-16$.

[21] Bin Z, Qiao W, Xiao-Liang C. Mode correlation and coherent-mode decomposition of laser beams. China Physics. 2003; 12:981-985.

[22] Baykal Y. Average transmittance in turbulence for partially coherent sources. Optics Communications. 2004; 231:129-136.

[23] Salem M, Shirai T, Dogariu A, Wolf E. Long-distance propagation of partially coherent beams through atmospheric turbulence. Optics Communications. 2003; 216:261-265.

[24] Ricklin J, Davidson F, Atmospheric optical communication with a Gaussian schell beam. Journal of the Optical Society of America. 2003; 20:856-866.

[25] Love G, Kirby A, Ramsey R. Sub-millisecond, high stroke phase modulation using polymer network liquid crystals. Optics Express. 2010; 18:7384-7389.

[26] Frigo M, Johnson S. Fftw: An adaptive software architecture for the fft. Proceedings of the International Conference on Acoustics, Speech, and Signal Processing. 1998; 3:1381-1384. 
[27] Banach V, Buldakov V, Mironov V. Intensity fluctuations of a partially coherent light beam in a turbulent atmosphere. Optical Spektrosk. 1983; 54:1054-1059.

[28] Voelz D, Fitzhenry K. Pseudo-partially coherent beam for free-space laser communication. Proceedings of SPIE. 2004; 5550:218-224.

[29] Verdeyen J. Laser Electronics. 3rd ed. Englewood Cliffs(NJ): Prentice Hall; 1995.

[30] Vaughn J. Atmospheric phase screens. Matlab Adaptive Optics Toolbox. 2002.

[31] Goodman J. Introduction to Fourier optics. 3rd ed. Englewood(CO): Roberts and Company; 2005.

[32] Lexitek Inc[Internet]. Wellesley(MA): Lexitek Inc. Pseudo-random phase plates. 2002. http://www.lexitek.com/pdfs/PhasePlatePaper.pdf

[33] Voelz D, Roggemann M. Digital simulation of scalar optical diffraction: revisiting chirp function sampling criteria and consequences. Applied Optics. 2009; $48(32)$.

[34] Lyke S, Voelz D, Roggemann M. Probability density of aperture-averaged irradiance fluctuations for long range free space optical communication links. Applied Optics. 2009; 48:6511-6527.

[35] Voelz D. Computational Fourier Optics: A MATLAB Tutorial. 1st ed. Bellingham(WA): SPIE Press; 2011.

[36] Schmidt J. Numerical Simulation of Optical Wave Propagation with Examples in MATLAB. Bellingham(WA): SPIE Press; 2010. 


\section{Index}

adaptive, 1, 3

amplitude, 12, 15, 37, 53

atmosphere, 7, 10, 11, 14, 18, 52, 56

Bernoulli, 3

coherence, 2, 4, 11, 12

coherent, 1, 7, 8

control, 8, 9, 47

controlled

coherence, 4

dB, 44-46

decibels, 44

decrease, 10, 12, 18, 23, 26

diffuser, 3

experiment, 15, 18-21, 25, 26, 47

far-field, 4, 9, 15, 17, 20, 25

FCS, 4, 34, 35, 41, 42, 44-46

field, 1, 11-13, 25, 26, 34, 55

free space optical, 7, 26, 34, 50

Fried, 18

FSO, 1, 5, 9, 25, 26, 38, 43, 58
Gaussian, 8, 9, 11, 13-15, 17, 18, 51, 52

Schell, 2, 4, 5, 8, 9, 34, 51

GSM, 2-4, 35, 40-49, 58, 59

increase, 15,59

intensity, 1, 22

Kelvin, 11

Kolmogorov, 19-22, 26, 36, 55

LASER, 1

liquid crystal, 8, 9, 25

maximum, 41, 42, 44-46, 53

mean, $8,10,13,15,18,34,35,40-43$

minimum, 9, 39, 41, 42, 44-46, 59

near-field, 9, 15

partial coherence, 8, 9, 25, 26

partially

coherent, 8, 26

spatial coherent, 26

PCB, 34, 35, 40, 43-45, 47

phase, $2,3,8,9,11,12,15,17,19-22$,

$25,26,36-38,51-56$ 
control, 8, 9, 51, 52, 54, 56

control screen, 51, 52

screen, 12, 15, 19-21, 36-38, 53, 55

pressure, 1, 10, 11

scintillation index, 10, 13, 18, 19, 23-26, $34,43,51,57,58$

screen, 15, 20-22

SI, 4

simulation, 14-16, 21, 35, 37-40, 55, 56

SLM, 3, 4, 19, 21-26, 35, 37, 52-54, 5659

spatial, 7-12, 14, 18, 19, 21, 23-31, 38, $41,44-46$

variance, 45,46

spatial correlation width, $12,34,35,38$, 47,53

spatial light modulator, 8, 25, 47, 51, 52, 56,59

speckle, 1 speckled, 23, 34

Speckles, 20

statistical, 12

statistics, 8, 9, 55

temperature, 1, 10, 11

turbulence, 4, 5, 22, 26, 34-36, 38, 40,
$42,45-47,53-55,57-59$

screen, 16, 18, 20, 55

UCL, 4, 38, 43-47

upper-coherence, 35, 40

variance, $7,10,13,18,23-25,34,35,41$, $42,44-46$ 